\title{
GALEX-SDSS-WISE LEGACY CATALOG (GSWLC): STAR FORMATION RATES, STELLAR MASSES, AND DUST ATTENUATIONS OF 700,000 LOW-REDSHIFT GALAXIES
}

\author{
Samir Salim ${ }^{1}$, Janice C. Lee ${ }^{2,3}$, Steven Janowiecki $^{4}$, Elisabete da Cunha ${ }^{5}$, Mark Dickinson ${ }^{6}$, Médéric Boquien ${ }^{7}$, \\ Denis Burgarella ${ }^{8}$, John J. Salzer ${ }^{1}$, and Stéphane Charlot ${ }^{9}$ \\ ${ }^{1}$ Department of Astronomy, Indiana University, Bloomington, IN 47404, USA; salims@ @indiana.edu \\ ${ }^{2}$ Space Telescope Science Institute, Baltimore, MD 21218, USA \\ ${ }^{3}$ Visiting Astronomer, Spitzer Science Center, Caltech, Pasadena, CA 91125, USA \\ ${ }^{4}$ International Centre for Radio Astronomy Research (ICRAR), The University of Western Australia, Perth, WA 6009, Australia \\ ${ }^{5}$ Research School of Astronomy and Astrophysics, Australian National University, Canberra, ACT 2611, Australia \\ ${ }^{6}$ National Optical Astronomy Observatory, Tucson, AZ 85719, USA \\ ${ }^{7}$ Universidad de Antofagasta, Unidad de Astronomía, Antofagasta 1270300, Chile \\ ${ }^{8}$ Laboratoire d'Astrophysique de Marseille, Aix-Marseille Universite, CNRS, F-13013 Marseille, France \\ ${ }^{9}$ Institut d'Astrophysique de Paris, CNRS, F-75014 Paris, France \\ Received 2016 August 22; revised 2016 September 28; accepted 2016 September 30; published 2016 November 9
}

\begin{abstract}
In this paper, we present the GALEX-SDSS-WISE Legacy Catalog (GSWLC), a catalog of physical properties (stellar masses, dust attenuations, and star formation rates [SFRs]) for $\sim 700,000$ galaxies with Sloan Digital Sky Survey (SDSS) redshifts below 0.3. GSWLC contains galaxies within the Galaxy Evolution Explorer footprint, regardless of a UV detection, covering $90 \%$ of SDSS. The physical properties were obtained from UV/optical spectral energy distribution (SED) fitting following Bayesian methodology of Salim et al., with improvements such as blending corrections for low-resolution UV photometry, flexible dust attenuation laws, and emission-line corrections. GSWLC also includes mid-IR SFRs derived from IR templates based on $22 \mu \mathrm{m}$ Wide-field Infrared Survey Explorer observations. These estimates are independent of UV/optical SED fitting, in order to separate possible systematics. The paper argues that the comparison of specific SFRs (sSFRs) is more informative and physically motivated than the comparison of SFRs. The sSFRs resulting from the UV/optical SED fitting are compared to the mid-IR sSFRs and to sSFRs from three published catalogs. For "main-sequence" galaxies with no active galactic nucleus (AGN) all sSFRs are in very good agreement (within 0.1 dex on average). In particular, the widely used aperture-corrected SFRs from the MPA/JHU catalog show no systematic offsets, in contrast to some integral field spectroscopy results. For galaxies below the main sequence (log sSFR $<-11)$, mid-IR (s)SFRs based on fixed luminosity-SFR conversion are severely biased (up to 2 dex) because the dust is primarily heated by old stars. Furthermore, mid-IR (s)SFRs are overestimated by up to 0.6 dex for galaxies with AGNs, presumably due to nonstellar dust heating. UV/optical (s)SFRs are thus preferred to IR-based (s)SFRs for quenched galaxies and those that host AGNs.
\end{abstract}

Key words: galaxies: fundamental parameters - galaxies: star formation

\section{INTRODUCTION}

The stellar mass and the current star formation rate (SFR) are two of the most fundamental physical properties of a galaxy. The SFR normalized by stellar mass, i.e., the specific star formation rate (sSFR; Bothun 1982; Tully et al. 1982), in addition serves as a rough indicator of a galaxy's star formation (SF) history. To infer properties such as stellar mass and SFR from observed quantities, stellar populations and the effects of dust attenuation are modeled and compared to galaxy spectra (e.g., Cid Fernandes et al. 2005) and/or the integrated photometry.

Pioneering work to derive physical properties of galaxies from their integrated light (e.g., Faber 1972; Searle et al. 1973; Tinsley \& Gunn 1976; Larson \& Tinsley 1978) paved the way for the development of more advanced stellar population synthesis (SPS) models in the 1980s (e.g., Bruzual 1983; Renzini \& Buzzoni 1986; Guiderdoni \& Rocca-Volmerange 1987). These conventional models were replaced by isochrone synthesis in the following decade (e.g., Charlot \& Bruzual 1991; Bruzual \& Charlot 1993), culminating in modern, high-resolution models (e.g., Bruzual \& Charlot 2003; Maraston 2005; Conroy et al. 2010b).
SPS models are the basis for widely used "simple" relations for inferring the stellar mass from broadband optical colors (e.g., Bell \& de Jong 2001; Courteau et al. 2014). These relations are by necessity averaged over galaxy populations and use simplified assumptions, so they cannot take into account variations in SF history, dust attenuation, or stellar metallicity. Simple relations are also used for inferring the SFR from UV or H $\alpha$ luminosity (Kennicutt 1998; Calzetti 2013, p. 419). In addition to again using average assumptions, these luminosities first need to be corrected for dust attenuation. For galaxies at $z \gtrsim 0$, the quantities that enter these relations must be explicitly corrected to rest-frame luminosities, which requires the spectral energy distribution (SED) of a galaxy to be assumed.

A more flexible and comprehensive approach is to derive physical parameters through SED fitting (see Walcher et al. 2011; Conroy 2013, for an overview). SPS models are used to produce a library (a grid, in the case of regular sampling intervals) of models in which the SF history and metallicity take a range of values. Model SEDs are further subjected to varying degrees of dust attenuation assuming some attenuation law. Redshifted model fluxes (broadband magnitudes) are compared to observations (obviating a need for an explicit 
$K$-correction), and the best-fitting model is sought and its parameters are adopted as the parameters of the observed galaxy. Unlike with simple relations, in SED fitting every observed flux plays some role in constraining all derived parameters, taking full advantage of the full set of observations. While in SED fitting the relationships between the observed and derived parameters are not explicit as with the simple relations, it should be noted that both approaches are ultimately rooted in SPS models.

For stellar SED fitting to produce useful constraints on the ongoing SFR, the rest-frame UV photometry must be included. SED fitting was initially employed to derive SFRs from broadband observations of small samples of Lyman break galaxies at $2<z<3$, for which the UV was redshifted to the easily observed optical range (Sawicki \& Yee 1998; Papovich et al. 2001; Shapley et al. 2005). Subsequently, the UV surveys carried out by the Galaxy Evolution Explorer (GALEX) satellite (Martin et al. 2005) placed low-redshift $(z<0.3)$ galaxies within the domain of SED fitting. Salim et al. (2005, 2007) performed the SED fitting of low-redshift galaxies by combining the UV photometry from GALEX with the optical photometry from Sloan Digital Sky Survey (SDSS). Furthermore, they utilized a Bayesian approach to SED fitting, following the methods pioneered by the Max Planck Institute for Astrophysics/Johns Hopkins University (MPA/JHU) group (Kauffmann et al. 2003b; Brinchmann et al. 2004; Tremonti et al. 2004). Unlike the traditional best-fit (minimum $\chi^{2}$ ) approach, Bayesian SED fitting determines the full probability distribution function (PDF) of any parameter (or combination of parameters), yielding more robust parameter characterization, along with its uncertainty (e.g., Taylor et al. 2011). Subsequent efforts expanded Bayesian SED fitting to include thermal dust emission (da Cunha et al. 2008; Noll et al. 2009) and star formation histories derived from cosmological simulations (Pacifici et al. 2013).

Salim et al. (2007, hereafter S07) performed a thorough investigation of SFRs in the local universe and showed that the UV/optical SED fitting is especially powerful for obtaining SFRs of galaxies with low sSFRs. However, S07 used an early GALEX data release covering only $10 \%$ of the SDSS area. Because of its preliminary nature, the catalog of physical parameters from S07 was not released to the public.

Here we present the GALEX-SDSS-WISE Legacy Catalog (GSWLC) of physical parameters, which builds on S07 UV/ optical SED fitting efforts, but includes numerous methodological improvements, such as UV photometry corrections, flexible dust attenuation curves, and emission-line corrections. Many of these improvements were made possible by the use of the Code Investigating GALaxy Emission ${ }^{10}$ (CIGALE; Noll et al. 2009, M. Boquien et al. 2016, in preparation) to calculate the models and perform the SED fitting. GSWLC contains SDSS galaxies within the GALEX footprint (regardless of the UV detection), covering $90 \%$ of the SDSS area. In addition to the stellar masses and the SFRs from the UV/optical SED fitting, GSWLC also includes SFRs derived independently from Wide-field Infrared Survey Explorer (WISE; Wright et al. 2010) mid-infrared (IR) observations. Community access to the catalog is provided online athttp://pages.iu.edu/ $\sim$ salims/gswlc and from the Mikulski Archive for Space Telescopes (MAST).

\footnotetext{
${ }^{10}$ http://cigale.lam.fr
}

After defining the scope and the sample of GSWLC (Section 2), we describe the input data and the process of deriving the parameters (Sections 3-5). The catalog is described in Section 6. In Section 7 we examine the contents of the catalog, while in Section 8 GSWLC is compared to several previously published catalogs of physical properties of SDSS galaxies.

\section{SAMPLE}

\subsection{Scope and SDSS Target Sample}

GSWLC is built around the SDSS Main Galaxy Sample (MGS; Strauss et al. 2002), the most extensive spectroscopic survey of the low-redshift $(z<0.3)$ universe. More specifically, GSWLC includes all MGS-like SDSS galaxies that fall within the GALEX footprint, covering 90\% of the SDSS area, or $\sim 8000 \mathrm{deg}^{2}$. Galaxies are kept in the sample regardless of the detection in the UV, thus retaining the optical selection. SDSS and GALEX data were obtained through the SDSS CasJobs SQL server.

The majority of low-redshift galaxies in SDSS were targeted spectroscopically as part of the magnitude-limited MGS, one of the three original ("legacy") spectroscopic surveys (the others being the luminous red galaxy [LRG] and quasar surveys). Legacy surveys were completed in 2008 with the release of DR7 (Abazajian et al. 2009) and cover a large contiguous area in the northern Galactic cap and three separate stripes in the southern Galactic cap (Figure 1). These areas lie almost entirely in the northern sky $\left(\delta>-10^{\circ}\right)$ and mostly at high Galactic latitude $\left(|b|>30^{\circ}\right)$.

The MGS selection algorithm targets nonstellar objects with well-measured photometry brighter than $r_{\text {petro }}=17.77$, with some additional cuts based on surface brightness (Strauss et al. 2002). The magnitude limit of MGS yields a sample of galaxies peaking at $z \sim 0.1$, with very few galaxies above $z=0.3$.

Selection of SDSS targets is performed on DR10 (Ahn et al. 2014), based on the SpecPhoto table, which combines primary (nonduplicate) photometric objects with primary spectroscopic observations. Nevertheless, in 25 cases more than one primary spectrum was found for the same photometric object, in which case we selected the spectrum that was closest to the photometric position, leaving 730,288 unique objects (objects with a unique ObjID). These SDSS targets constitute shaded areas in Figure 1.

Our selection for inclusion in GSWLC consists of only two criteria:

$$
\begin{aligned}
& r_{\text {petro }}<18.0 \\
& 0.01<z<0.30
\end{aligned}
$$

The first criterion replicates the MGS brightness cut, with some rounding to allow for targeting photometry fluctuations. The second cut removes stars and very nearby galaxies whose photometry is likely to be less accurate because of their large angular size (West et al. 2010), and whose redshifts are poorer indications of the distance (Tully et al. 2016). GSWLC therefore includes the entire MGS, complemented by MGSlike galaxies from other programs that fall within the brightness (and, effectively, the redshift) cut of MGS: $5.2 \%$ from BOSS (typically ellipticals), and the remaining $2.1 \%$ from other surveys (QSO, SEGUE). For studies that focus on the general 

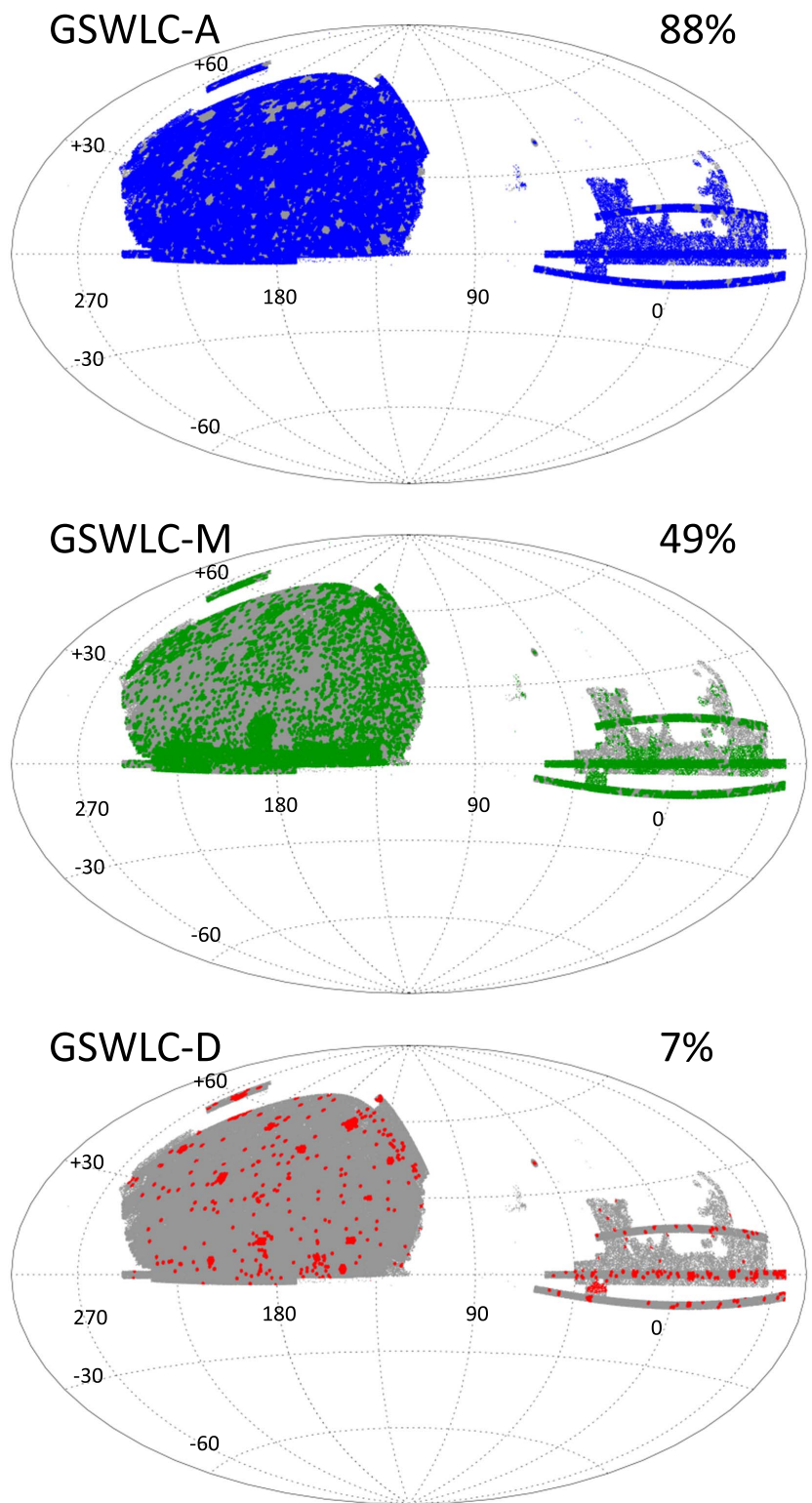

Figure 1. Distribution on the sky of galaxies included in GSWLC. Each panel shows SDSS target galaxies as shaded areas, covering $\sim 9000 \mathrm{deg}^{2}$. The SDSS MGS legacy survey area consists of the contiguous area on the left (northern Galactic cap) and three horizontal stripes on the right. Nonstripe areas on the right are primarily from the SDSS BOSS survey of passive galaxies. BOSS galaxies are also interspersed in the contiguous area on the left. Superimposed on each panel in color are SDSS galaxies that fall within the coverage of one of the GALEX imaging surveys: the shallow (all-sky) survey (AIS), the mediumdeep survey (MIS), or the deep survey (DIS), thus defining the coverage of GSWLC-A, M, and D. The percentage of SDSS targets covered by each GALEX survey is indicated in the upper right corner of each panel.

population of galaxies, it is strongly recommended to use only the MGS galaxies (flag_mgs $=1$ in GSWLC).

\subsection{The Final Sample}

GSWLC includes all SDSS targets within areas covered by $G A L E X$, regardless of a UV detection. GALEX has observed the sky in 1.2 . wide circular fields ("tiles"), with a wide range of exposure times (Morrissey et al. 2007). The greatest UV sky coverage is provided by shallow $(\sim 100 \mathrm{~s})$ observations, most of which were taken as part of the All-sky Imaging Survey
(AIS). GALEX could not safely point in the direction of bright stars, so even the AIS's coverage contains holes. Observations of medium depth, corresponding to one GALEX orbit ( $\sim 1500 \mathrm{~s})$, are the basis for the Medium Imaging Survey (MIS), which specifically targeted areas within the SDSS footprint. For select fields, deeper observations were obtained by co-adding the observations from multiple orbits, to produce the Deep Imaging Survey (DIS), with nominal exposure time of 30,000 s.

Since the accuracy of UV photometry, and consequently of the derived physical properties, will depend on the depth of the observations, we produce three separate catalogs, each approximately corresponding to shallow (all-sky; A), medium (M), and deep (D) UV imaging surveys. The catalogs are designated as GSWLC-A, GSWLC-M, and GSWLC-D. Each catalog is based only on GALEX imaging of a certain depth, even if deeper exposures of an object exist:

$$
\begin{array}{ll}
\text { GSWLC-A: } & t_{\mathrm{NUV}} \leqslant 650 \\
\text { GSWLC-M: } & 650<t_{\mathrm{NUV}}<4000 \\
\text { GSWLC-D: } & t_{\mathrm{NUV}} \geqslant 4000,
\end{array}
$$

where the exposure times in near-UV (NUV) band are given in seconds. Far-UV (FUV) exposure times are typically identical to NUV times (the two bands were observed simultaneously), except in cases when the FUV image was missing due to camera malfunction. Note that in GSWLC-M we also include individual visits used for DIS co-adds, since the exposure times of individual visits fall in the range for GSWLC-M.

To define the final samples for inclusion in the catalogs, we take all SDSS targets from Section 2.1 that fall within 0.6 of the GALEX tile centers of the specific UV survey (A, M, or D). This gives the following final sample sizes:

$$
\begin{array}{ll}
\text { GSWLC-A: } & 640,659(88 \%) \\
\text { GSWLC-M: } & 361,328(49 \%) \\
\text { GSWLC-D: } & 48,401(7 \%)
\end{array}
$$

where the percentage indicates the fraction of all SDSS DR10 targets. GSWLC-M contains $7 \times$ as many galaxies as the MIS sample used in S07. Since GALEX surveys are nested, objects in the deeper catalogs are mostly included in the shallower ones. The number of unique galaxies encompassed by the three catalogs, 658,911, is therefore only slightly larger than the number of objects in GSWLC-A and corresponds to $90 \%$ of the SDSS target sample.

\subsection{SDSS-GALEX Matching}

GALEX data are taken from the final data release (GR6/7). ${ }^{11}$ Matching of SDSS to GALEX is in general a nontrivial task (Budavári et al. 2009) because of the changes in galaxy morphology with wavelength and different resolutions ( $5^{\prime \prime}$ for GALEX versus 1". 3 for SDSS). Furthermore, the GALEX data release contains detections of the same objects from multiple tiles. Thus, one needs to address the cases when multiple UV candidates exist for an optical source, and when the UV source may be a blend of two or more optical sources.

In our case, the problem of SDSS-GALEX matching is significantly alleviated by the fact that it involves relatively bright objects, having low sky density. In particular, we find that there are essentially no cases of genuine multiple UV

\footnotetext{
${ }^{11}$ http://galex.stsci.edu/GR6/
} 


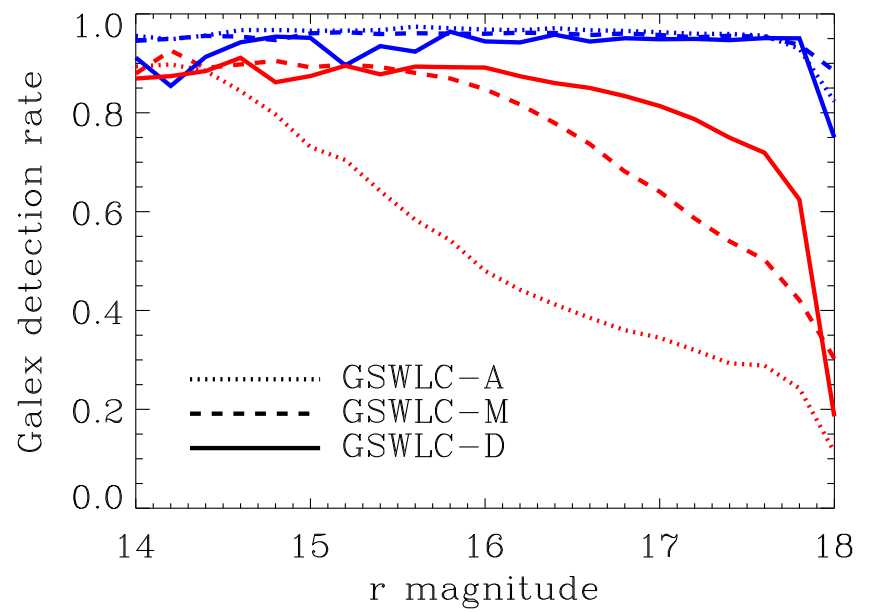

Figure 2. UV detection rates of different GSWLC catalogs (defined by UV survey depth). Detection rate is shown as a function of $r$ magnitude, separately for blue ( $g-r<0.7$, observer frame; blue lines) and red galaxies (red lines). The detection rate of red galaxies is strongly dependent on UV depth and magnitude.

candidates within the $5^{\prime \prime}$ search radius. The choice of search radius is based on GALEX positions of isolated SDSS targets, for which we find negligible bulk offset with respect to SDSS ( 0 .! 2 in either R.A. or decl.) and $1 \sigma 1 \mathrm{D}$ positional uncertainty of 1." 3 . When multiple UV candidates are nominally present, they are either due to multiple observations (overlapping tiles) or from NUV and FUV detections of the same object that were erroneously left as separate sources in the GALEX merged-band catalog. When there are multiple observations of the same object, we take the one from the tile with the longest FUV exposure (if no candidate has an available FUV image, the tile with the longest NUV exposure is selected).

While each SDSS object has an unambiguous GALEX match, the UV measurement may still be affected by blending of several objects independently detected in SDSS. We will address this issue and describe the implemented solution in Section 3.

UV detection rates of SDSS objects ( $3 \sigma$ threshold, either UV band) are 54\% for GSWLC-A, 74\% for GSWLC-M, and 84\% for GSWLC-D. Besides the UV survey depth, the detection rates depend strongly on the galaxy's sSFR or color, as can be seen from Figure 2, where we plot the detection rates as a function of $r$-band magnitude, separately for blue $(g-r<0.7)$ and red galaxies.

\subsection{SDSS-WISE and 2MASS Matching}

GSWLC utilizes WISE observations at $22 \mu \mathrm{m}$ (WISE channel W4) to determine SFRs independently from the UV/ optical SED fitting. The depth of WISE observations over the sky is not uniform ( $6 \pm 1 \mathrm{mag}$ Vega), but it is still much more uniform than the GALEX depth and essentially covers the entire sky without gaps. The FWHM of the W4 PSF is $12^{\prime \prime}$. We use $22 \mu \mathrm{m}$ photometry from two independent reductions of WISE data. The first is the official AllWISE Source Catalog, ${ }^{12}$ hosted at the IPAC Infrared Science Archive (IRSA) at the Caltech/ JPL, and the second is the "unWISE" reduction (Lang et al. 2016), ${ }^{13}$ where SDSS detections served as forced photometry priors.

\footnotetext{
12 http://wise2.ipac.caltech.edu/docs/release/allwise/

13 http://www.unwise.me
}

To match SDSS to AllWISE, we take the closest candidate in a $5^{\prime \prime}$ search radius. Note that AllWISE detections and astrometry are based on simultaneous PSF-matched fits to all channels. Thus, the shorter-wavelength channels (3.4 (W1) and $4.6 \mu \mathrm{m}$ (W2)), which have smaller PSF widths $\left(6^{\prime \prime}\right)$ and higher signal-to-noise ratio $(\mathrm{S} / \mathrm{N})$, will dominate the astrometric accuracy $(<0,5)$. This makes the adopted search radius sufficient to capture genuine matches and justifies taking the closer candidate in the case of multiple matches $3.2 \%$ of cases). The detection rate of SDSS targets at $22 \mu \mathrm{m}$ (based on the $2 \sigma$ threshold of profile magnitudes) is $32 \%$, which is lower than the detection rate of GALEX.

Since the unWISE catalog is derived based on SDSS DR10 detections, no matching is required (sources already carry SDSS ObjID). Forced photometry increases the $\mathrm{S} / \mathrm{N}$ of measured fluxes, so the $2 \sigma$ detection rate is $41 \%$, one-third higher than that of the official catalog.

Near-IR photometry $\left(J H K_{s}\right)$ is taken from the Two Micron All Sky Survey (2MASS) Extended Source Catalog (XSC), also hosted at IRSA. We match SDSS to 2MASS XSC by selecting the closest candidate within the $5^{\prime \prime}$ search radius. The detection rate of SDSS targets is $48 \%$.

\section{DATA}

The data used to construct GSWLC consist of broadband photometry (magnitudes or fluxes and their errors) from GALEX, SDSS, and WISE and redshifts from SDSS. Comparison data sets will also use line fluxes from SDSS spectra.

\subsection{Optical Photometry}

SDSS offers several choices for galaxy photometry: modelMag (magnitude extracted assuming either a de Vaucouleurs or an exponential profile), cmodelMag (weighted average of de Vaucouleurs and exponential magnitudes), and petroMag (surface-brightness-dependent aperture magnitude). The usual practice in SED fitting is to use fluxes that yield the most accurate colors. Of SDSS magnitudes, ModelMag best fulfills that role. We confirm that this is the optimal choice by finding that the best-fitting models (based on optical fitting alone) have three times lower median $\chi^{2}$ when modelMag is used as opposed to cmodelMag or petroMag. Also, sSFRs obtained from UV/optical SED fitting that uses modelMag magnitudes have a smaller scatter with respect to sSFRs from WISE and from $\mathrm{H} \alpha$. While ModelMag produces stable colors, the degree to which it will estimate the total light will depend on galaxy size, morphology (Bernardi et al. 2010; Taylor et al. 2011), and color gradient. Simard et al. (2011) and Meert et al. (2016) have derived magnitudes that were designed to better capture the total light in each band. Readers can use those catalogs to scale our estimates of the stellar mass and $\mathrm{SFR}$, if that is required for their goals. Based on the comparison of ModelMag and Meert et al. (2016) $r$-band magnitudes, the typical correction should nevertheless be relatively modest $(+0.03 \pm 0.08 \mathrm{dex})$.

\subsection{UV Photometry and Corrections}

The GALEX source catalog offers only one measure of flux that is recommended for galaxies: MAG_AUTO, a Kron elliptical aperture magnitude derived by SExtractor (Bertin \& Arnouts 1996). We investigate the differences in the methodology of this measurement compared to the one used for SDSS 
photometry, as well as the effects of lower GALEX resolution, by deriving MAG_AUTO magnitudes of 1000 randomly selected SDSS targets whose $g$-band images were degraded to match GALEX resolution. By comparison with isolated sources, we find that the differences between $G A L E X$-resolution MAG_AUTO and SDSS-resolution modelMag are dominated by systematic offsets (up to $0.4 \mathrm{mag}$ ) arising from close companions (especially at $d<10^{\prime \prime}$ ) that become blended in lower resolution, rather than the differences in the methodology of magnitude measurement ( $<0.1$ mag difference).

To account for the systematics that arise from both the difference in GALEX and SDSS resolutions and the methods of flux measurement, we derive and implement several corrections to UV fluxes. Corrections are derived by comparing the UV magnitudes predicted from optical-only SED fitting of starforming galaxies $(g-r<0.7)$ with the actual UV magnitudes. While any individual predicted UV magnitude is crude $(\sigma \approx 0.4 \mathrm{mag}$ in NUV), the corrections are accurate owing to a very large number of galaxies that define them.

First, we confirm that there are no zero-point offsets between the predicted and the actual UV magnitudes. Next, we find that a small edge-of-detector correction is required for NUV, but not FUV, photometry. NUV magnitudes of sources that appear in the outermost $8^{\prime}$ annulus of the field of view, which accounts for $40 \%$ of the detector area, are up to $0.1 \mathrm{mag}$ too bright, possibly because of an inaccurate flat fielding. The amount of correction depends linearly on the radial position within the annulus:

$$
\mathrm{NUV}_{\text {corr }}=\mathrm{NUV}+0.78 \mathrm{FOV}-0.37, \quad \mathrm{FOV}>0.47
$$

where FOV is the distance from the center of the tile in degrees.

Next, we derive the centroid shift correction. The shift between optical and UV positions arises due to random errors, mostly from the lower accuracy of the GALEX astrometry, but also from the differences in UV versus optical morphology, which have a slight effect on the measured magnitudes. Again, using the difference between predicted and observed NUV magnitudes, we find the following correction:

$$
\mathrm{UV}_{\text {corr }}=\mathrm{UV}+0.054 \Delta x-0.049, \quad \Delta x>0.7
$$

where $\Delta x$ is the shift between GALEX and SDSS positions in arcseconds. A correction is not applied when $\Delta x \leqslant 0$ !! 7 , where random errors dominate. We apply the same correction to NUV and FUV magnitudes.

By far the most significant correction for UV magnitudes is due to blending. The degree to which a GALEX magnitude will be affected by blending will depend on both the relative brightness of the nearest companion (any SDSS photometric object) and its separation $(d)$ from the target. We construct corrections (same for NUV and FUV) as a function of the difference in $g$ magnitude $\left(\Delta g=g_{\text {comp }}-g_{\text {target }}\right)$ for four ranges of separation:

$$
\begin{array}{ll}
\mathrm{UV}_{\text {corr }}=\mathrm{UV}-0.036 \Delta g+0.37, & d<4^{\prime \prime} \\
\mathrm{UV}_{\text {corr }}=\mathrm{UV}-0.046 \Delta g+0.35, & 4^{\prime \prime} \leqslant d<10^{\prime \prime} \\
\mathrm{UV}_{\text {corr }}=\mathrm{UV}-0.019 \Delta g+0.15, & 10^{\prime \prime} \leqslant d<15^{\prime \prime} \\
\mathrm{UV}_{\text {corr }}=\mathrm{UV}-0.006 \Delta g+0.04, & 15^{\prime \prime} \leqslant d<20^{\prime \prime} .
\end{array}
$$

We note that even though the corrections to UV magnitudes may be significant for individual objects (in particular those with a close blended companion), the SFRs for the majority of star-forming galaxies are not significantly affected by their application.

We use FUV fluxes measured at NUV positions (FUV_N$\mathrm{CAT}$ ) rather than from independent FUV detections, as they provide more robust UV color. Both NUV and FUV measurements require a $3 \sigma$ threshold in FLUX_AUTO.

\subsection{Galactic Reddening and Calibration Errors}

SDSS and GALEX photometry must be corrected for galactic reddening. We find that the combination of extinction coefficients from Peek \& Schiminovich (2013) for UV bands and Yuan et al. (2013) for optical bands produces somewhat better fits $\left(\sim 10 \%\right.$ smaller $\left.\chi^{2}\right)$ than the corrections based on Schlegel et al. (1998) or on Yuan et al. (2013) alone. We reproduce the adopted corrections here:

$$
\begin{aligned}
A_{\mathrm{FUV}}= & 10.47 E(B-V)+8.59 E(B-V)^{2} \\
& -82.8 E(B-V)^{3} \\
A_{\mathrm{NUV}}= & 8.36 E(B-V)+14.3 E(B-V)^{2} \\
& -82.8 E(B-V)^{3} \\
A_{u}= & 4.39 E(B-V) \\
A_{g}= & 3.30 E(B-V) \\
A_{r}= & 2.31 E(B-V) \\
A_{i}= & 1.71 E(B-V) \\
A_{z}= & 1.29 E(B-V) .
\end{aligned}
$$

Photometry catalogs usually list only random flux errors, without systematic or calibration errors. We find that the default CIGALE padding of formal photometry errors by the addition (in quadrature) of $0.1 \mathrm{mag}$ to catalog magnitude errors to account for systematic errors in models and photometry leads to a significant loss of derived physical parameter accuracy, as evidenced by $50 \%$ larger scatter when derived SFRs are compared with independent SFR estimates. Instead, following S07, we add more modest calibration errors (determined from repeat observations) of $(0.052,0.026,0.02$, $0.01,0.01,0.01,0.01) \mathrm{mag}$ in (FUV, NUV, $u, g, r, i, z)$, plus the $u$-band red leak error of $\sigma_{u, \mathrm{RL}}=0.0865(r-i)^{2}+0.0679(r-i)$, derived in S07 based on the description of the red leak in Abazajian et al. (2004). The validity of the adopted calibration errors is verified by constructing the distribution of magnitude residuals (fitted minus real magnitude divided by the total error), which follow unit Gaussians.

\subsection{Mid-IR Photometry and Ancillary Data}

We derive what we refer to as "mid-IR SFRs" using two types of $22 \mu \mathrm{m}$ magnitude (flux) measurements. The first is what the AllWISE Source Catalog calls a profile-fitting magnitude (w4mpro). The profile is simply the PSF, so these magnitudes are essentially PSF magnitudes and are thus most appropriate for unresolved sources. Since the PSF FWHM at $22 \mu \mathrm{m}$ is $12^{\prime \prime}$, which is larger than $91 \%$ of galaxies ( $r_{90}$ size), these magnitudes are reasonably appropriate for SDSS galaxies. Yet, PSF magnitudes will systematically underestimate the flux in larger galaxies. This is largely remedied in forced photometry from the unWISE catalog, which applies SDSS-measured galaxy profiles (convoluted with W4 PSF) as photometry priors.

To derive emission-line SFRs (which we call "H $\alpha$ SFRs"), we use $\mathrm{H} \alpha$ and $\mathrm{H} \beta$ fluxes from the MPA/JHU catalog, based 
on DR7. ${ }^{14}$ Measurement of emission-line fluxes is described in Tremonti et al. (2004). We also use the emission-line (BPT diagram; Baldwin et al. 1981) classification of galaxies from the MPA/JHU catalog, derived as described in Brinchmann et al. (2004, hereafter B04).

\section{DERIVATION OF MID-IR AND EMISSION-LINE SFRs}

Although CIGALE allows the IR dust emission to be fit in conjunction with the stellar emission, we derive the mid-IR SFRs separately, to avoid potential systematics. For example, CIGALE normalizes the IR SED template, so that the total dust emission (i.e., total IR luminosity, $L_{\mathrm{IR}}$ ) equals the stellar emission absorbed by the dust in the UV/optical/near-IR. However, the shape of the IR SED is a free parameter (Noll et al. 2009), which means that without the far-IR SED, as in the case of WISE data, the IR luminosity will not be strongly constrained and could thus be driven, through energy balance requirement, by any potential systematics in the estimate of the absorbed stellar luminosity, such as those that would arise from incorrect assumptions about the attenuation law.

Instead, accurate IR luminosities can be obtained from $22 \mu \mathrm{m}$ observations using luminosity-dependent IR templates, and these estimates can then be compared to the results of the SED fitting of the stellar emission (for actively star-forming galaxies, where dust heating by young stars dominates), to verify whether energy balance is satisfied.

Another advantage of performing the stellar SED fitting separately is that in a joint fit to stellar and dust emission one also has to worry about the contamination of IR emission from dust-obscured type 2 AGNs (such as Seyfert 2, or highexcitation radio galaxies; Yan et al. 2013; Pace \& Salim 2016). While the AGN contribution can be modeled in CIGALE, it introduces additional degrees of freedom, further weakening the usefulness of an energy balance requirement, in particular when only mid-IR photometry is available.

The mid-IR SFRs that we report in GSWLC are computed as follows. First, we calculate the total IR luminosity (8-1000 $\mu \mathrm{m}$; Sanders \& Mirabel 1996) by interpolating the luminosity-dependent IR templates of Chary \& Elbaz (2001) so that they match the $22 \mu \mathrm{m}$ flux. We also tested using Dale \& Helou (2002) templates, which do not have an associated luminosity, but the IR SED shape-luminosity dependence is imposed from empirically calibrated relations of Marcillac et al. (2006). The two methods produce very similar IR luminosities (average difference of 0.01 dex, scatter of 0.02 dex), but Chary $\&$ Elbaz (2001) based luminosities are marginally $(<2 \%)$ better correlated with IR luminosities from Herschel, so we adopt them for GSWLC.

We check the robustness of our $22 \mu \mathrm{m}$ derived IR luminosities by comparing them to IR luminosities derived for SDSS Stripe82 galaxies by Rosario et al. (2016). Their IR luminosity is obtained by fitting IR templates to WISE $22 \mu \mathrm{m}$ flux and the submillimeter fluxes from Herschel-SPIRE (250, 350 , and $500 \mu \mathrm{m})$. Because they have multiple flux points, they derive IR luminosities from Dale \& Helou (2002) IR templates that do not have an associated luminosity. The cross-matched sample consists of 817 galaxies. As can be seen in Figure 3, the agreement of IR luminosities is excellent, with no $(<0.01 \mathrm{dex})$ systematic offset over the range probed by Herschel

\footnotetext{
$\overline{14 \text { http://www.mpa-garching.mpg.de/SDSS }}$ DR7;

http://home.strw.leidenuniv.nl/ jarle/SDSS/
}

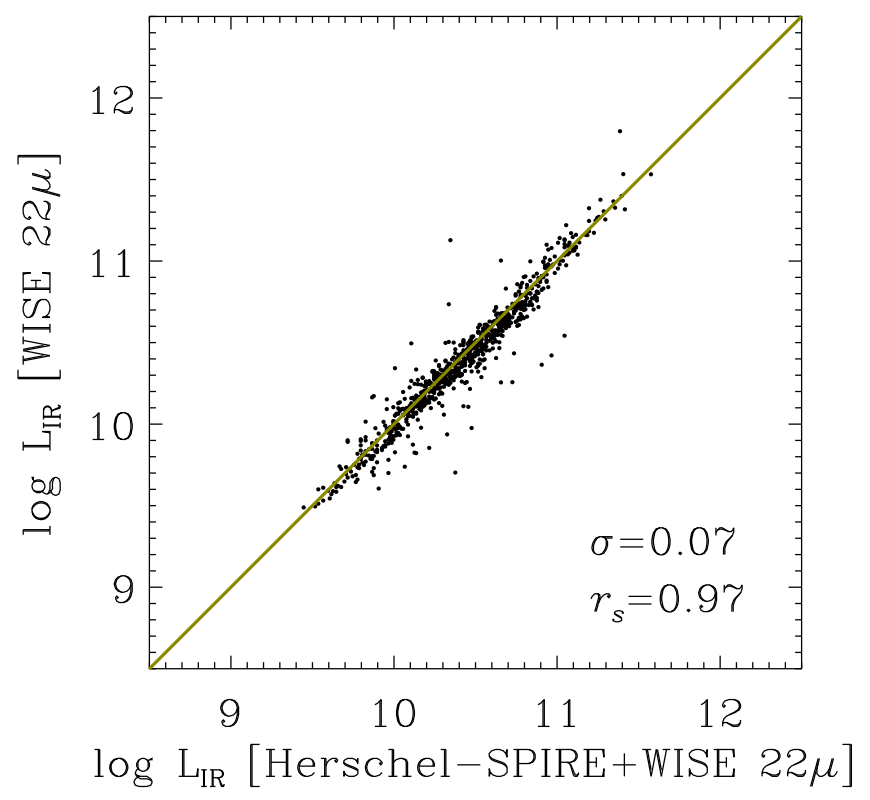

Figure 3. Comparison of IR luminosities derived from WISE $22 \mu \mathrm{m}$ flux, against Herschel + WISE IR luminosities from Rosario et al. (2016). Our WISE IR luminosity is based on a single flux point in the mid-IR, converted to total IR luminosity using the luminosity-dependent IR templates of Chary \& Elbaz (2001), whereas the Herschel + WISE IR luminosity comes from $22 \mu \mathrm{m}$ and submillimeter $(250-500 \mu \mathrm{m})$ measurements. The two estimates agree very well. The solid line is a $1: 1$ relation.

$\left(9.5<\log L_{\mathrm{IR}}<11.4\right)$, perfect linearity, and a scatter of only 0.07 dex. Comparison of the specific luminosities $\left(L_{\mathrm{IR}} / M_{*}\right)$, which removes the distance dependence (Section 7), reveals a mild nonlinearity (with a slope of 1.10), in the sense that WISE IR luminosities of galaxies with the lowest specific luminosity (or sSFR) are 0.1 dex lower compared to galaxies with the highest specific luminosity. While the agreement between our and the Rosario et al. IR luminosities is encouraging, there is a caveat that the latter are not fully independent from our estimates, because both use $22 \mu \mathrm{m}$ flux. The role of the $22 \mu \mathrm{m}$ flux point in the Rosario et al. estimate is likely significant given that their data do not sample the peak of the IR SED $(\sim 100 \mu \mathrm{m})$.

The above comparison was carried out for $22 \mu \mathrm{m}$ IR luminosities derived using profile (PSF) fluxes from the official AllWISE catalog. If we instead used $22 \mu \mathrm{m}$ photometry from unWISE (Lang et al. 2016), the small scatter with respect to Rosario et al. (2016) Herschel + WISE IR luminosities of $\sigma=0.07$ dex (Figure 3) would grow to $\sigma=0.14$. This does not necessarily imply that unWISE fluxes are noisier, because Herschel + WISE uses the PSF flux from the AllWISE catalog.

To obtain mid-IR SFRs from IR luminosity, we use a simple conversion given by Kennicutt (1998), adjusted to the Chabrier IMF using the 1.58 conversion factor (S07):

$$
\log \mathrm{SFR}=\log L_{\mathrm{IR}}-9.966
$$

where $\mathrm{SFR}$ is in $M_{\odot} \mathrm{yr}^{-1}$ and $L_{\mathrm{IR}}$ in $L_{\odot}$.

To derive $\mathrm{H} \alpha$ SFRs, which we use for comparison purposes, we take $\mathrm{H} \alpha$ fluxes and correct them using the Balmer decrement method, assuming case $\mathrm{B}$ recombination and the Cardelli et al. (1989) extinction curve. Emission-line fluxes are measured within $3^{\prime \prime}$ SDSS fibers, which we find to capture, on average, $30 \%$ of the galaxy mass, depending on the galaxy's distance, size, and profile. Dust-corrected $\mathrm{H} \alpha$ fluxes are then 
converted to SFR using the Kennicutt (1998) relation, adjusted by -0.24 dex (Muzzin et al. 2010) to convert from the Salpeter to Chabrier IMF. Comparison of the fiber $\mathrm{H} \alpha \mathrm{SFR}$ with other, total SFR indicators can be accomplished if all measurements are converted to sSFRs. The $\mathrm{H} \alpha$ sSFR is obtained by normalizing by the stellar mass present within the fiber, which are taken from the MPA/JHU catalog. ${ }^{15}$

\section{DERIVATION OF PHYSICAL PARAMETERS FROM UV/OPTICAL SED FITTING}

\subsection{Methodology}

Physical parameters from UV/optical SEDs are derived using CIGALE (Noll et al. 2009; M. Boquien et al. 2016, in preparation), a powerful code that produces libraries (grids) of model SEDs and performs the SED fitting. The code was originally written in Fortran and recently rewritten in Python. It is continually being improved and expanded in capabilities. We use the most recent Python version 0.9, which offers full control over parameters that specify SF history, dust attenuation, and emission-line fluxes. This flexibility has proved essential in order to derive robust results.

Model SEDs produced by CIGALE, and from which model photometry is extracted, consist of UV/optical/near-IR stellar emission $(\lambda \lesssim 5 \mu \mathrm{m})$ and, optionally, the IR dust emission $(\lambda \gtrsim 5 \mu \mathrm{m})$. A third, also optional component is the nebular emission (lines and continuum). For reasons discussed in Section 4, we will restrict the SED fitting to stellar emission, with the contribution of emission lines included.

CIGALE synthesizes stellar emission based on either Bruzual \& Charlot (2003, BC03) or Maraston (2005, M05) SPS. The most important difference between them is in the treatment of the TP-AGB phase of stellar evolution (Maraston 2011; Marigo 2015). CIGALE cannot currently include the contribution of emission lines in conjunction with M05 models, which, as we will demonstrate, are important for obtaining robust measurements. Therefore, we opt to use $\mathrm{BC} 03$ models. The use of $\mathrm{BC} 03$ models may anyhow be a more appropriate choice, given some evidence that $\mathrm{BC} 03$ models better reproduce optical and near-IR colors than M05 models (Conroy \& Gunn 2010a; Kriek et al. 2010; Zibetti et al. 2013).

CIGALE can also perform Bayesian SED fitting and report the physical parameters and their errors. Bayesian SED fitting consists of building PDFs of physical parameters by assigning probabilities to each model spectrum (at a matching redshift) based on the goodness of fit between the model and observed broadband SEDs. The methodology has been described in detail in S07, da Cunha et al. (2008), and other papers. Walcher et al. (2011) and Conroy (2013) provide comprehensive reviews of various aspects of SED fitting. While SED fitting represents an optimal way to extract information from photometry (and/or spectra), it will be limited by the uncertainties in stellar and dust attenuation models (Conroy et al. 2009, 2010b; Conroy \& Gunn 2010a; Mitchell et al. 2013) and the choice of priors (e.g., appropriate SF histories and dust attenuation laws).

In order to specify the modeling assumptions (e.g., which dust model or parameterization of SF history to use), the usual practice is to select the parameters that yield the smallest relative differences between the observed and the best-fitting

\footnotetext{
15 Stellar masses and SFRs from the MPA/JHU catalog have been adjusted from the Kroupa to Chabrier IMF by applying a -0.025 dex offset (S07).
}

model photometry, i.e., that minimize some average $\chi^{2}$ of the sample. However, in some cases different modeling assumptions may lead to marginal changes in $\chi^{2}$ (suggesting that UV/ optical SED is degenerate with respect to them). We will therefore require both the internal consistency $\left(\right.$ small $\left.\chi^{2}\right)$ and the external one: maximum correlation of the SED fitting SFR with respect to two entirely independently derived SFR tracers (mid-IR and $\mathrm{H} \alpha$ ).

In our analysis we assume a Chabrier IMF (Chabrier 2003). CIGALE assumes a flat WMAP7 cosmology $\left(H_{0}=70 \mathrm{~km} \mathrm{~s}^{-1}\right.$ $\left.\mathrm{Mpc}^{-1}, \Omega_{m}=0.27\right)$.

\subsection{Near-IR Photometry}

We perform SED fitting on UV and optical photometry $(0.15-0.9 \mu \mathrm{m})$, omitting the longer-wavelength stellar emission $(1.1-5.4 \mu \mathrm{m})$. The near-IR photometry is excluded because it increases the reduced $\chi^{2}$ values and worsens (albeit slightly) the correlations with mid-IR and $\mathrm{H} \alpha$-derived SFRs. Parameters from the SED fitting are reliable to the extent that the models are able to reproduce the observed colors. We find that the model photometry, based on either $\mathrm{BC} 03$ or $\mathrm{M} 05$, does not accurately reproduce the near-IR colors from 2MASS photometry. In particular, both models imply a strong correlation between $J-K$ and $i-J$, which is not seen in the data ( $\mathrm{S}$. Salim 2016, in preparation).

Stellar mass estimates based on near-IR photometry are often perceived as more accurate and/or more precise than those based on optical bands, or that, at the minimum, the near-IR photometry improves the precision of stellar mass estimates. The reasoning underlying this claim is that stellar emission peaks in the near-IR and that near-IR mass-to-light ratio is less sensitive to the stellar population age and the effects of dust. While these arguments are correct, they do not take into account large modeling discrepancies in the near-IR mass-tolight ratios (McGaugh \& Schombert 2014), arising due to the uncertainties in our understanding of the post-main-sequence phases of stellar evolution, the phases that dominate the energy output in the near-IR (Conroy 2013). For example, van der Wel et al. (2006) find that the inclusion of near-IR photometry in SED fits leads to discrepancies with respect to dynamical masses, and that these discrepancies depend on the SPS model used.

Even if there existed no major uncertainties in the models and no discrepancies between the models and the data (thus, no concerns that the near-IR will affect the accuracy of mass estimates), the improvement in the precision of stellar masses achieved by adding the near-IR photometry to the optical photometry is much more modest than usually assumed. Taylor et al. (2011) have studied physical parameters derived from mock observations of GAMA galaxies when just the optical photometry, or optical plus UKIDSS-depth near-IR photometry, was used to perform the SED fitting. They show that the improvement in the precision of stellar masses when the nearIR photometry is added to SED fitting is small (0.05 versus 0.06 dex; their Figure A2). The parameter whose precision improves the most is the stellar metallicity. Indeed, the sensitivity of near-IR luminosities, and even red optical bands ( $i$ or $z$ ), to metallicity (e.g., Figure 9 of Courteau et al. 2014; Figure 10 of Taylor et al. 2011) is another reason why near-IR alone is a more problematic tracer of stellar mass than the multiband optical light. 


\subsection{Star Formation Histories}

In the following three sections we discuss the choice of modeling parameters, starting with SF histories and metallicities.

BC03 models are available for six stellar metallicities, of which we use the higher four, from 0.2 to $2.5 Z_{\odot}$, which is an adequate range for most galaxies in SDSS (Gallazzi et al. 2005).

We have considered two different parameterizations of SF history offered by CIGALE: two-component exponential and delayed exponential. In the two-component exponential model, the SF history is a composite of two exponentially declining functions ( $\tau$ models), each with its own starting epoch (age) and $e$-folding time. The normalization of the younger population is specified by the mass fraction $f$, which can also be set to zero, resulting in a single exponential model. Each component starts at maximum value and then decreases monotonically. In contrast, the delayed exponential SF history is smooth (e.g., Gavazzi et al. 2002): it starts from zero SFR, reaches a peak at some time, and then declines. It is given by

$$
\mathrm{SFR} \propto \frac{t}{\tau^{2}} e^{-t / \tau}
$$

We find that as long as the parameters are chosen so that the model colors (and more specifically, model sSFRs) cover the range of colors or sSFRs present in observations (for delayed SFHs this requires allowing $\tau<0$ in Equation (9)), either SF history will produce similar stellar masses and SFRs. Specifically, there is no systematic difference for masses of non-main-sequence, low-sSFR galaxies (log sSFR <-11), while the difference in masses for actively star-forming galaxies is typically 0.1 dex. The difference in SFRs is similar in degree (see also Boquien et al. 2014) and of the same sign, which means that the difference in sSFRs is less than the difference in either mass or SFR. While both parameterizations perform reasonably well, the two-component exponential SF histories yield better fits (geometric mean of reduced $\chi^{2}$ of 0.7 versus 1.0), presumably because, by not being smooth, they are able to better match the bursty SF history of low-mass galaxies (Weisz et al. 2011). We thus adopt the two-component exponential parameterization.

Delayed exponential models have been preferred over exponential models in some recent studies (e.g., Simha et al. 2014). However, there are important differences among the exponential models. If a single-exponential model is assumed to have started in the early universe (e.g., $t_{0} \sim 10^{10} \mathrm{yr}$ ), in order to acknowledge the fact that galaxies contain ancient stellar populations no matter how dominant the current episode of SF may be (Aloisi et al. 2007), then such a model will fail to reproduce the sSFRs of many star-forming galaxies today, simply because $\log \mathrm{SSFR}_{\max }=\log t_{0}^{-1}=-10$. It is this naive implementation of the exponential model (e.g., Simha et al. 2014) that results in inferior performance. The problem of the single-exponential model not producing high sSFRs can be alleviated by allowing the starting epoch of the model to be more recent than the big bang. Though later-epoch galaxy formation is obviously not realistic, it can instead be interpreted as the epoch of peak of SF activity. Variable starting time was assumed in essentially all work that based their SF histories on a single exponential, including S07, which in addition had stochastic bursts superimposed on exponential models.
Allowing recent starting times for the exponential model results in SFRs that are comparable to more sophisticated models. ${ }^{16}$ However, this approach will neglect ("outshine") any old population, resulting in somewhat underestimated masses (Papovich et al. 2001; Michałowski et al. 2014). This problem is resolved with a two-component exponential model used here, where one component corresponds to high-redshift SF. While more realistic SF histories may have multiple bursts, or are in general quite variable (Weisz et al. 2011), modeling these features with high temporal precision is unimportant from the standpoint of broadband SED fitting, where even the characteristics of the most recent burst cannot be constrained with any precision because of the burst age versus burst amplitude degeneracy (e.g., Smith \& Hayward 2015).

The parameters of the adopted two-component exponential model are as follows. The formation time of the old population is $10 \mathrm{Gyr}$ before the observation epoch, with $e$-folding times that span a range from $850 \mathrm{Myr}$ (fast decline that defines the lower sSFR limit of $\log \mathrm{sSFR}=-13.8$ ) to a nearly constant $20 \mathrm{Gyr}$ (which has declined only $0.2 \mathrm{dex}$ since formation). Formation times for the younger component span from $100 \mathrm{Myr}$ (the shortest timescale to which UV observations are sensitive) to $5 \mathrm{Gyr}$. Their $e$-folding time is $20 \mathrm{Gyr}$, i.e., nearly constant. In order to produce the highest sSFRs observed today (log sSFR $\sim-8$ ), the mass fraction of the younger component must span up to $f=0.5$. Thus, our library of SF histories looks like old exponentials with various decay times, with a relatively flat burst superimposed.

\subsection{Dust Attenuation Laws}

CIGALE allows the dust attenuation law to be specified as a simple power law $\left(k(\lambda)_{\mathrm{PL}} \propto \lambda^{\alpha}\right.$, following Charlot \& Fall 2000), or according to the Calzetti et al. (2000) recipe $\left(k(\lambda)_{\text {Cal }}\right)$. The Calzetti curve can be modified so that the overall slope becomes more or less steep, by multiplying it by the power law of slope $\delta$ (Noll et al. 2009):

$$
k(\lambda)_{\bmod }=k(\lambda)_{\mathrm{Cal}}(\lambda / 5500 \AA)^{\delta} .
$$

Note that slope modification changes $R_{V}$ from 4.05 , the value it has for the Calzetti curve. The attenuation law can be further modified to include a UV bump of varying strength (Stecher 1965; Fitzpatrick \& Massa 1986; Conroy et al. 2010a).

We systematically tested various modifications of the Calzetti attenuation law. We produce model grids where the range of color indices lies between $E(B-V)=0.1$ and $0.6 \mathrm{mag}$ for the young $(<10 \mathrm{Myr})$ population (i.e., the dust affecting the nebular lines; equivalent to $A_{\mathrm{H} \alpha}=0.25-1.5 \mathrm{mag}$ ). Attenuation that affects older stars, and therefore most of the stellar continuum, is taken to be smaller by a factor of 0.44 , following Calzetti et al. (2000). The tests were carried out with galaxies classified in the BPT diagram as star-forming, for which mid-IR luminosity and dust-corrected $\mathrm{H} \alpha$ luminosity provide reliable estimates of SFR. The results are illustrated in Figure 4. The upper panels show the comparison between sSFR

\footnotetext{
16 Pacifici et al. (2015) report large $(0.6$ dex, on average) offsets at $z>1$ between SFRs derived using their classical models (which assume exponential histories with a range of formation times) and their more sophisticated models (SF history based on cosmological simulations). However, the two models also assume different attenuation laws (fixed slope versus multiple slopes), which will affect the SFRs through the derived dust attenuation (see their Figure 6), so it is not clear that the difference in SF histories is responsible for most of the offset.
} 
obtained from the SED fitting, assuming the standard Calzetti curve, which has no UV bump, and independently derived midIR and $\mathrm{H} \alpha$ sSFRs. Note that the comparison is carried out in terms of sSFR, which better reveals systematic trends than the usual SFR comparison (Section 7). Similar trends are seen versus mid-IR and $\mathrm{H} \alpha$. The differences increase with the increasing sSFR until $\log \mathrm{sSFR}_{\mathrm{SED}}=-9$, in the sense that the values derived from SED fitting assuming the Calzetti curve tend to be up to 0.4 dex higher. At still higher sSFRs, however, there is reasonable agreement between the sSFRs, as expected, since the Calzetti curve was derived from starburst galaxies with high sSFRs.

The middle row shows the comparisons when the Calzetti law is modified to include a UV bump of varying intensity (from no bump to $4 \times$ the Milky Way [MW] value). The UV/ optical SED fits become formally better (geometric mean of reduced $\chi^{2}$ s goes from 1.3 to 0.8 ), and the correlation with mid-IR and $\mathrm{H} \alpha$ sSFRs improves (from $\sigma=0.40$ to 0.32 dex, for mid-IR comparison). However, the nonlinearity (the slope of the correlation featuring logarithms of sSFRs not being unity) persists, as well as a tail of galaxies with unusually low sSFRs.

The bottom row shows the comparisons when the Calzetti attenuation law is further modified to make it steeper (with $\delta=-0.5$ and -1.0 ), in addition to allowing the UV bump. There is now a significant reduction in scatter with respect to mid-IR and $\mathrm{H} \alpha$ sSFRs $(\sigma=0.23)$, and the relation is fairly linear. This is our adopted dust attenuation model. We note that achieving this level of agreement between SED-fitting sSFRs and mid-IR sSFRs requires both the steepening of the attenuation curve and the addition of the UV bump. Just steepening the slope of the attenuation curve does remove most of the nonlinearity with respect to mid-IR sSFR (plot not shown), but still yields relatively high reduced $\chi^{2}$ of 1.0 , regardless of the amount of steepening. In other words, the combination of the two modifications is necessary to achieve both the small $\chi^{2}$ values and good agreement with other indicators.

The agreement between SED and mid-IR SFRs demonstrates that the energy balance is fulfilled when this modified attenuation law is used: the energy absorbed by the dust in the stellar SED matches the energy emitted in the IR.

We note that assuming an attenuation curve in a power-law form, having slopes $\alpha=-1.0$ (preferred by the majority of galaxies) and $\alpha=-1.5$, and adding the UV bump to them has a similar effect to modifying the standard Calzetti curve as described in the preceding paragraph. For comparison, the standard Calzetti curve can be approximated in the UV range by a power law of slope $\alpha=-0.5$.

It must be stressed that the differences in sSFR obtained with various attenuation laws are largely driven by the changes in SFR, rather than $M_{*}$. The stellar masses of star-forming galaxies obtained with the Calzetti dust attenuation law are on average only 0.06 dex lower than the stellar masses obtained with the modified law. However, the masses of a small number of individual galaxies, especially those with very high sSFR, can differ up to 0.4 dex in either direction, i.e., the dispersion of the two mass estimates increases with sSFR. For completeness, we report that for passive galaxies the use of the Calzetti dust law yields stellar masses that are 0.06 dex higher than the ones obtained with our modified attenuation law, and this difference is rather constant from one passive galaxy to another, i.e., it has a small dispersion of only 0.02 dex.

We conclude that while star-forming galaxies may be governed by a range of attenuation laws, they generally exhibit a steeper law than the standard Calzetti curve $(\delta=-0.6$, on average) and include a UV bump (1.1 times the MW value, on average). This average curve has $R_{V} \approx 2.5$, compared with $R_{V}=4.05$ for the Calzetti curve, and agrees well with the curve derived empirically by Conroy et al. (2010a): an MWlike curve with $R_{V}=2.0$ instead of $R_{V}=3.1$ (reducing $R_{V}$ makes the curve steeper). Evidence for a steeper-than-Calzetti curve has been found in other studies as well (Charlot \& Fall 2000; Buat et al. 2011; Hao et al. 2011; Wild et al. 2011; Salmon et al. 2015), though not as steep as the one found here and in Conroy et al. More detailed investigation of these results and their implications will be presented in a separate paper.

\subsection{Correction for Emission Lines}

Finally, we describe how we account for the flux from emission lines, which can have a significant effect on broadband fluxes and colors of galaxies with high equivalent widths (Papovich et al. 2001; Kauffmann et al. 2003b; Pacifici et al. 2015). CIGALE calculates the contribution of 124 lines, specified by metallicity (taken to equal the stellar metallicity of SPS models) and ionization parameter. We select the lowest ionization parameter available, $\log U=-3$, as it produces the biggest improvements and is closest to direct measurements (Dopita et al. 2000; Liang et al. 2006).

The formal quality of UV/optical fits is significantly improved by correcting for emission-line flux (geometric mean of $\chi_{\text {red }}^{2}$ drops from 1.2 to 0.7$)$. More importantly, accounting for emission lines significantly improves the correlation of SED sSFRs with other indicators. For galaxies with high sSFRs, not correcting for emission lines produces offsets of up to $0.5 \mathrm{dex}$, as can be seen by comparing Figure 5, which does not correct for emission lines, with the lower row of Figure 4. Emissionline correction makes very little difference for stellar masses, which change by $<0.01$ dex on average.

While the addition of emission lines to model fluxes results in overall improvement, the correction is not perfect. Nebular metallicity is fixed to stellar metallicity, which is not realistic. Also, the ionization parameter of many galaxies is lower than the minimum ionization parameter available in CIGALE. We see some redshift-dependent trends in SFR, as lines straddle across the bandpasses. We account for this systematic offset $(\sim 0.1$ dex on average) by deriving an average correction, in 0.01-wide bins of redshift, with respect to SFRs from B04 (using galaxies classified as star-forming; Section 8.2), and applying it to all SFRs from the SED fitting. We use B04 SFRs for this correction instead of mid-IR SFRs, because the latter are available for only $63 \%$ of star-forming galaxies. Nevertheless, the correction would have been essentially identical if mid-IR SFRs were used.

\subsection{Derived Parameters}

In summary, for each 0.01 -wide redshift bin, from 0.01 to 0.30 , we calculate a grid of 342,720 models, a factor-of-3 increase in the number of models and a factor-of-5 increase in redshift resolution with respect to what was used in S07. 


\section{Calzetti atten. curve + no UV bump $\left(\chi^{2}=1.3\right)$}

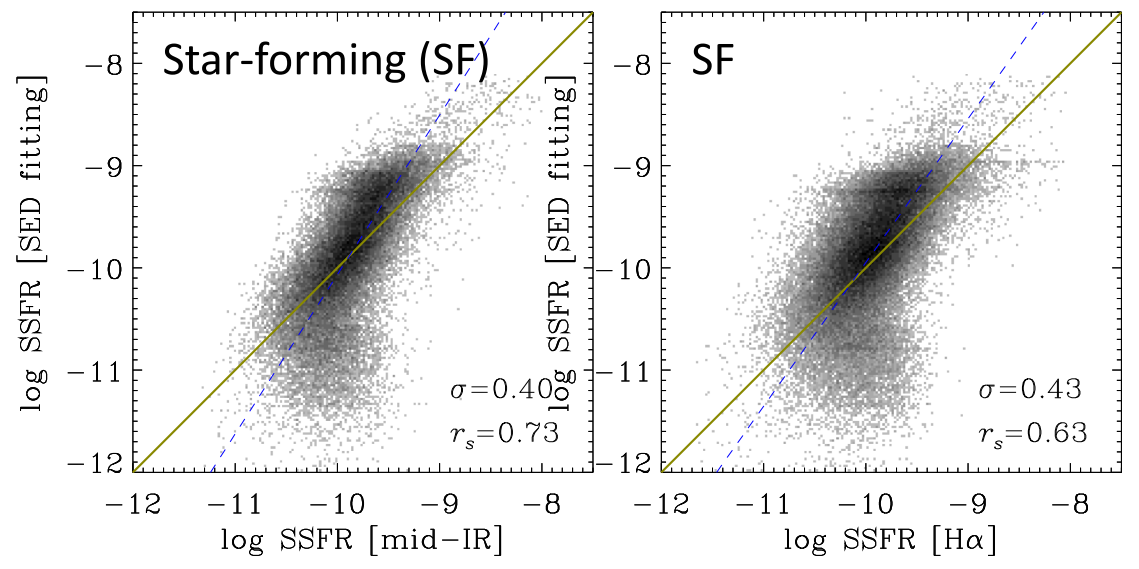

Calzetti atten. curve + UV bump allowed $\left(\chi^{2}=0.8\right)$

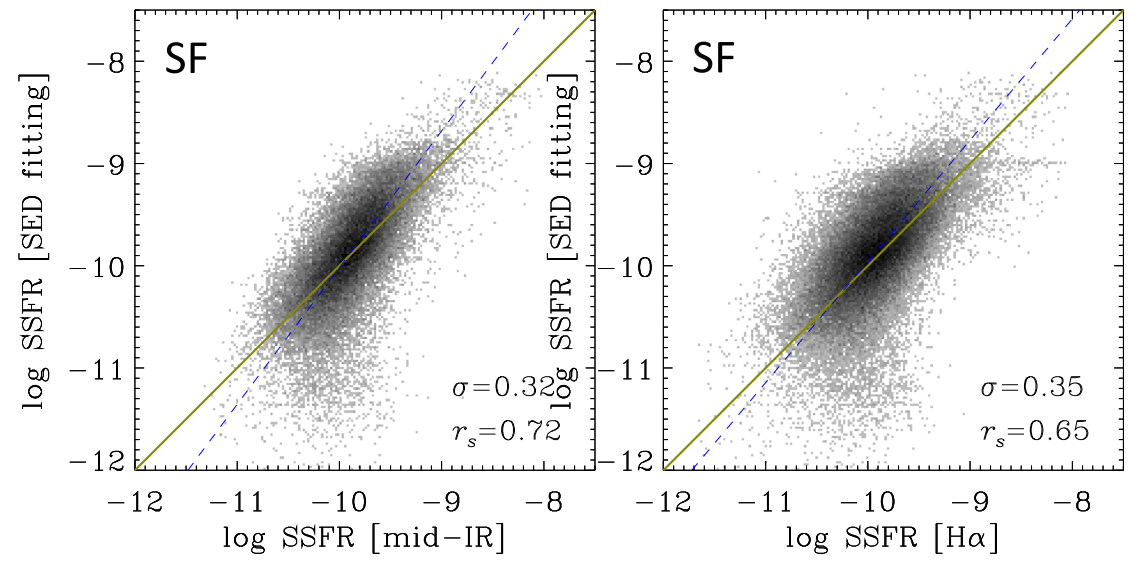

Modified atten. curve + UV bump $[=$ ADOPTED $]\left(\chi^{2}=0.7\right)$

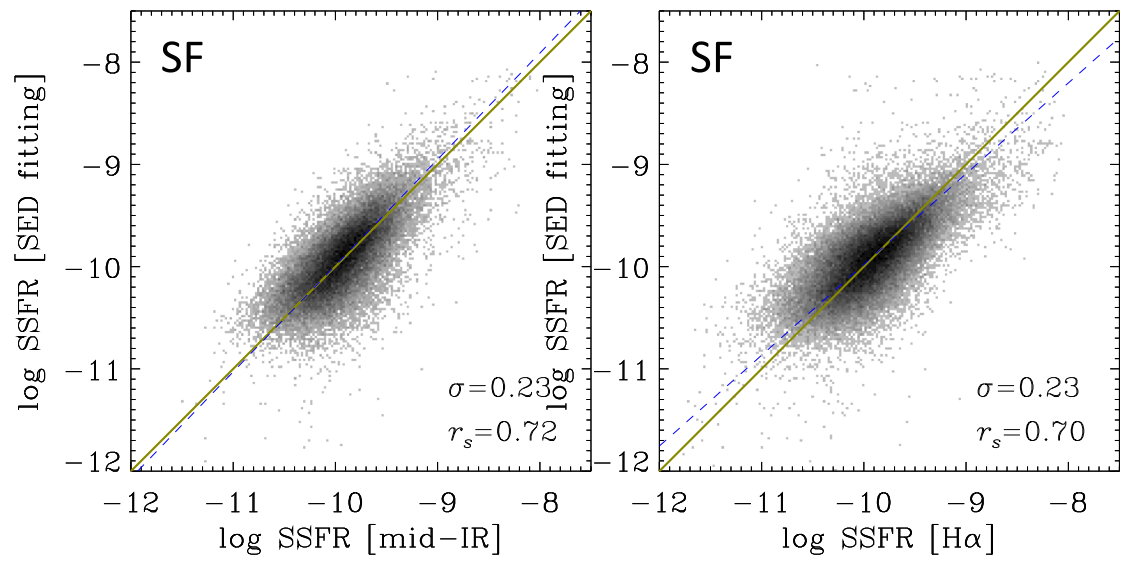

Figure 4. Choice of attenuation law to use in UV/optical SED fitting. We show a comparison between sSFRs obtained from the UV/optical SED fitting (assuming different attenuation laws) and sSFRs based on WISE $22 \mu \mathrm{m}$ observations (mid-IR sSFRs; left panels) and from Balmer-decrement-corrected H $\alpha$ (right panels). The upper panels use the standard Calzetti attenuation curve. Strong trends against both SFR tracers are seen. They are reduced by allowing for a UV bump (the middle row), which also improves the formal $\chi^{2}$ of SED fits. Further improvement, resulting in very good match with mid-IR and H $\alpha$ sSFRs, is achieved by also making the attenuation curve steeper than the Calzetti one (bottom row). The changes in sSFRs are mostly driven by the changes in SFRs, rather than the stellar masses. The solid line is a 1:1 relation, and the dashed line is the robust bisector linear fit (used throughout the paper). Standard deviation around the fit and the Spearman correlation coefficient are given in each panel. For these and subsequent figures (except where noted) the lower-redshift bound is 0.025 in order to reduce AllWISE photometry systematics for galaxies having large angular size. In order to present a large number of data points without blotting, we use gray scale where the shade scales as the number of galaxies per pixel to the power of 0.3. A sublinear exponent is chosen to better show the outliers. Shown are the data from GSWLC-M, but similar results are obtained with GSWLC-A or D.

From the SED fitting we report the logarithm of the current stellar mass $\left(M_{*}\right)$, the logarithm of the SFR averaged over the past $100 \mathrm{Myr}$ (the timescale for UV emission), and dust attenuations in FUV, $B$, and $V$ rest-frame bands. For each of these parameters CIGALE builds a PDF. The nominal value of the reported parameter is the average of the PDF. We have 


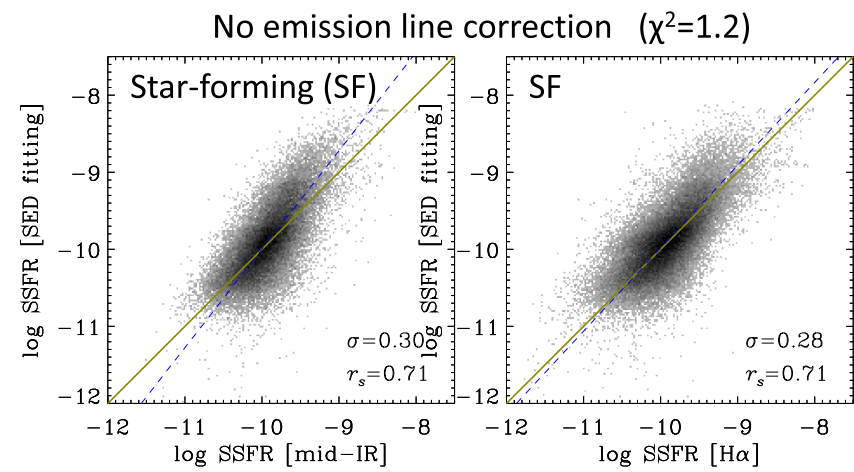

Figure 5. Effect on sSFRs of not correcting for nebular emission lines. This figure should be compared to the lower panels of Figure 4, where otherwiseidentical models include the contribution of emission lines. Not correcting for emission lines produces overestimates of SED (s)SFRs of high-sSFR galaxies, greater random errors (quantified as the standard deviation around the fit; blue dashed line), and also the increased mean reduced $\chi^{2}$.

performed mock fitting, in which true parameters are known, and find that the average of the PDF better retrieves the parameters than the median, and much better than the parameter corresponding to the best-fitting model. The bestfitting parameters are volatile and suffer from grid discreteness (e.g., Taylor et al. 2011). The formal error of the parameter is taken as the second moment (standard deviation) of the PDF. By default CIGALE applies a minimum error in the case in which the error from the PDF is lower than it, but we have disabled this adjustment. Instead, we caution the reader that the robustness of the error estimated from the PDF will depend on the goodness of the fit, i.e., it will be most accurate when $\chi_{r}^{2} \sim 1$.

\section{GSWLC}

In Table 1, we describe the contents of the catalog, including various IDs, coordinates, the SED-fitting parameters (SFR, $M_{*}$, and dust attenuations in several bands), mid-IR SFRs, and flags. Flags describe cases when the SED parameters or the mid-IR SFRs are not listed, as we discuss further below.

To review, the criteria for inclusion in the catalog are for an SDSS object to be covered by GALEX observations of a certain depth, regardless of whether it was detected in the UV. Furthermore, galaxies need to lie in the $0.01<z<0.30$ redshift range and be above the $r_{\text {petro }}=18.0$ magnitude threshold.

Approximately $1 \%$ of SDSS targets have spectra classified as "quasars" (CLASS = 1), based on the Balmer emission lines being broad. These galaxies are therefore likely to be type 1 AGNs, which include Seyfert 1s and, less frequently, true quasars. In type $1 \mathrm{AGNs}$ the accretion disk is not entirely obscured and can contribute to the UV/optical continuum of the host, which may significantly bias the derived SED parameters, especially the SFR. This is confirmed by the finding that the geometric mean of the reduced $\chi^{2}$ values of the best-fitting model of broad emission line galaxies is 4 times higher than that of other galaxies. We flag such objects in the final catalog and do not report their SED-fitting parameters. Type 1 AGNs are not obscured by a dusty torus, which is why they mostly contribute to the UV/optical SED. We thus leave their mid-IR SFRs in the catalog, but they should still be used with caution in case there is some AGN emission in the mid-IR.
We produce three separate catalogs, GSWLC-A, M, and D, according to the UV depth, as described in Section 2.2. In addition, we produce a master catalog that combines the three catalogs, keeping for each galaxy the data from the deepest catalog. This catalog is designated GSWLC-X and contains 658,911 objects. Readers are cautioned that nonuniform depth may lead to systematics in SFRs, especially for galaxies with $\log \mathrm{SSFR}_{\mathrm{SED}}<-11$, where the UV detection rate and the resulting quality differ from survey to survey (Figure 6). On the other hand, the stellar masses will not be subject to any such biases. All catalogs have the same format as described in Table 1.

The catalogs are currently publicly available at http://pages. iu.edu/ salims/gswlc, which will document any changes implemented in subsequent versions. The catalog letter designation is followed by a version number. The analyses in this paper are all based on Version 1 catalogs (e.g., GSWLCM1). GSWLC is also hosted at MAST and may be included in the SDSS SciServer and/or hosted by NED/IPAC in the future.

In Figure 6 we show mean random errors of $\log$ SFR $_{\mathrm{SED}}$ and $\log M_{*}$ as a function of sSFR, for each of the three catalogs GSWLC-A, M, and D. Errors in SFR depend very strongly on the sSFR, and for more passive galaxies also on the UV depth. The decrease of errors at the lowest and highest sSFRs is an artifact of reaching the boundary of the model grid. For passive galaxies the formal SFR errors range between 0.65 and 0.80 dex. However, the SFR error of passive galaxies that have no current SF whatsoever $(\log$ SSFR $<-\infty)$ is infinity (in $\log$ ). SFR values for galaxies with $\log$ sSFR $<-11.7$ $(<-11.5$ for GSWLC-A; $<-12.0$ for GSWLC-D) should be considered upper limits. For actively star-forming galaxies the SFR errors are typically below $0.1 \mathrm{dex}$, a $50 \%$ improvement over S07. Errors on stellar mass are typically much lower than the SFR errors, with the opposite dependence on SSFR, and little difference between the UV surveys. They range from $0.03 \mathrm{dex}$ for the passive galaxies to $0.10 \mathrm{dex}$ for the most active ones.

The geometric mean of the reduced $\chi^{2}$ values for the GSWLC-M catalog is 0.7 . However, the tail extends to $\chi_{r}^{2}$ values in excess of 100 . Instead of introducing an arbitrary cut to exclude poorly fit objects, we test the robustness of the derived SFRs and stellar masses as a function of the reduced $\chi^{2}$. We find that no systematic differences in excess of $0.1 \mathrm{dex}$ (with respect to mid-IR SFRs and stellar masses from B04) are present when $\chi_{r}^{2} \leqslant 30$. For those galaxies we retain the physical parameters from the SED fitting, and we annul them if $\chi_{r}^{2}>30(0.7 \%)$. Nevertheless, the readers are advised to treat galaxies with $\chi_{r}^{2} \geqslant 5$ with caution, especially for individual galaxies.

\section{COMPARISON BETWEEN sSFRs FROM THE SED FITTING, MID-IR, AND H $\alpha$ EMISSION}

In this section we discuss comparisons between various SFRs derived in this work: specifically, the UV/optical sSFRs from the SED fitting on the one side, and mid-IR and $\mathrm{H} \alpha$ sSFRs on the other. We remind the reader that the SED (s)SFRs are averaged over the preceding $100 \mathrm{Myr}$, the UV emission timescale.

The paper breaks away from the usual practice of comparing absolute SFRs and instead performs the comparison in terms of the specific SFRs. The standard SFR comparison has its merits 
Table 1

Contents of GALEX-SDSS-WISE Legacy Catalog (GSWLC)

\begin{tabular}{|c|c|c|c|}
\hline Column Number & Column Name & Units & Description \\
\hline 1 & ObjID & $\cdots$ & SDSS photometric identification number \\
\hline 2 & GLXID & $\ldots$ & GALEX photometric identification number \\
\hline 3 & plate & $\ldots$ & SDSS spectroscopic plate number \\
\hline 4 & MJD & $\cdots$ & SDSS spectroscopic plate date \\
\hline 5 & fiber ID & $\cdots$ & SDSS spectroscopic fiber identification number \\
\hline 6 & R.A. & degree & Right Ascension from SDSS \\
\hline 7 & Decl. & degree & Declination from SDSS \\
\hline 8 & $z$ & $\cdots$ & Redshift from SDSS \\
\hline 9 & $\chi_{r}^{2}$ & $\cdots$ & Reduced goodnes-of-fit value for the SED fitting \\
\hline 10 & $\log M_{*}$ & $M_{\odot}$ & Stellar mass \\
\hline 11 & $\sigma\left(\log M_{*}\right)$ & $M_{\odot}$ & Error of the stellar mass \\
\hline 12 & $\log$ SFRSED & $M_{\odot} \mathrm{yr}^{-1}$ & UV/optical (SED) SFR \\
\hline 13 & $\sigma\left(\log \mathrm{SFR}_{S E D}\right)$ & $M_{\odot} \mathrm{yr}^{-1}$ & Error of the SFR \\
\hline 14 & $A_{\mathrm{FUV}}$ & mag & Dust attenuation in rest-frame FUV \\
\hline 15 & $\sigma\left(A_{\mathrm{FUV}}\right)$ & mag & Error of dust attenuation in FUV \\
\hline 16 & $A_{B}$ & mag & Dust attenuation in rest-frame $B$ \\
\hline 17 & $\sigma\left(A_{B}\right)$ & mag & Error of dust attenuation in $B$ \\
\hline 18 & $A_{V}$ & mag & Dust attenuation in rest-frame $V$ \\
\hline 19 & $\sigma\left(A_{V}\right)$ & mag & Error of dust attenuation in $V$ \\
\hline 20 & flag_sed & $\cdots$ & SED-fitting flag $\left(0=\mathrm{OK}, 1=\right.$ broad-line spectrum, $2=\chi_{r}^{2}>30,5=$ missing SDSS photometry $)$ \\
\hline 21 & UV survey & $\cdots$ & $1=$ GSWLC-A, $2=$ GSWLC-M, $3=$ GSWLC-D \\
\hline 22 & $\log \mathrm{SFR}_{\text {mid }-\mathrm{IR}, \mathrm{AW}}$ & $M_{\odot} \mathrm{yr}^{-1}$ & Mid-IR SFR from WISE (AllWISE catalog) \\
\hline 23 & flag_wise & $\cdots$ & Mid-IR SFR (AllWISE) flag $(0=\mathrm{OK}, 1=$ no mid-IR SFR [low sSFR], $5=$ no $22 \mu$ m detection $)$ \\
\hline 24 & $\log \mathrm{SFR}_{\text {mid-IR,uW }}$ & $M_{\odot} \mathrm{yr}^{-1}$ & Mid-IR SFR from WISE (unWISE catalog) \\
\hline 25 & flag_unwise & $\cdots$ & Mid-IR SFR (unWISE) flag $(0=$ OK, $1=$ no mid-IR SFR [low sSFR], $5=$ no $22 \mu$ m detection $)$ \\
\hline 26 & flag_mgs & $\cdots$ & $0=$ not in SDSS MGS, $1=$ in MGS \\
\hline
\end{tabular}

Note. Columns (10)-(19) originate from the SED fitting. If there are multiple reasons for setting the flag, the flag value wil be the sum of individual flag values. When the SED (or un/wise) flag is set, the SED-fitting parameters (or mid-IR SFR) are not given. Mid-SFRs based on unWISE are recommended over the AllWISE ones for $z<0.06$ samples, large $\left(r>10^{\prime \prime}\right)$ galaxies, or studies that explore dependence of SFR on galaxy size or shape. SFRs and stellar masses are based on the Chabrier IMF. Missing values are listed as -99 .

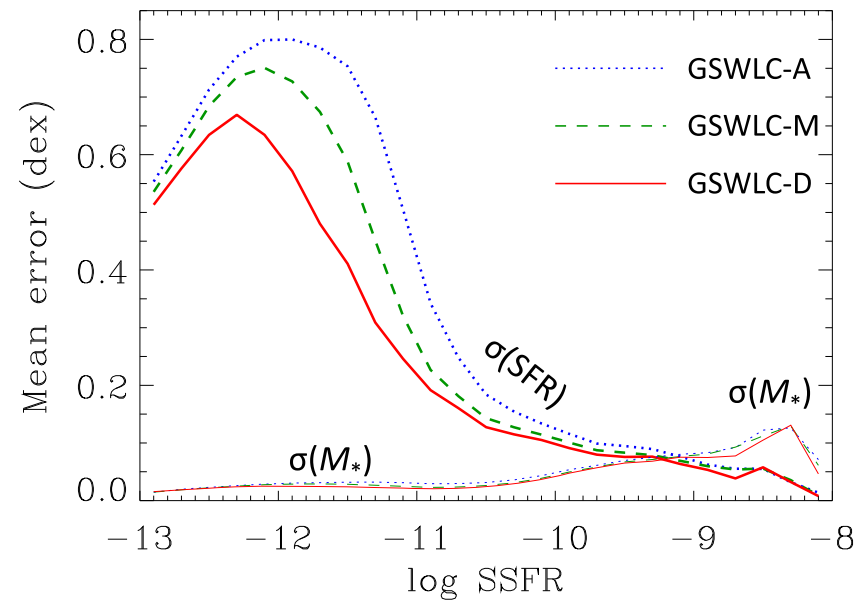

Figure 6. Mean random error of SED-fitting SFR (thick lines) and stellar mass (thin lines) as a function of sSFR and UV survey depth. The second moment of the parameter probability distribution function is adopted as the error of these parameters.

and may be more intuitive; however, when the focus is on galaxies with similar stellar populations, subject to similar systematics, the sSFR comparison has its advantages: (1) SDSS probes a large dynamic range of distances and therefore of luminosities, which means that SFRs (and stellar masses) will be correlated even if $\mathrm{SFR} / L\left(M_{*} / L\right)$ are not. This "trivial" dependence is eliminated in SSFR. For example, the correlation coefficient between SED and mid-IR SFRs (of galaxies classified as star-forming) is $r_{s}=0.86$, while it is $r_{s}=0.72$ for comparisons involving sSFRs. Consequently, the SFR comparison is seemingly tighter. (2) The comparison of sSFRs is more informative because it contrasts galaxies that are physically similar. For example, a galaxy with $\mathrm{SFR}=10 M_{\odot} \mathrm{yr}^{-1}$ can be a massive galaxy on the starforming sequence, or a lower-mass galaxy experiencing a starburst. However, their sSFRs will be different. Or, a galaxy with SFR $=0.01 M_{\odot} \mathrm{yr}^{-1}$ can be a blue star-forming dwarf, or a massive red galaxy, but their sSFRs will differ by two orders of magnitude. Finally, (3) the comparison in terms of sSFR allows for a meaningful comparison between total and fiber SF, as long as the fiber SFR is normalized by the stellar mass within the fiber.

In Figure 7 we present the comparison of UV/optical sSFRs (i.e., SED-fitting sSFRs) against mid-IR sSFRs from WISE $22 \mu \mathrm{m}$ photometry from AllWISE. We show the comparisons using the medium UV depth catalog (GSWLC$\mathrm{M}$ ), which balances the extensiveness of GSWLC-A with the depth of GSWLC-D. All of the findings hold for the more accurate GSWLC-D as well. We refrain from using GSWLC-X because of its nonuniform UV depth. The comparisons are presented for galaxies split into three categories using the BPT diagram (iclass in the MPA/JHU catalog). The star-forming class (iclass $=1$ ) includes galaxies with well-measured 

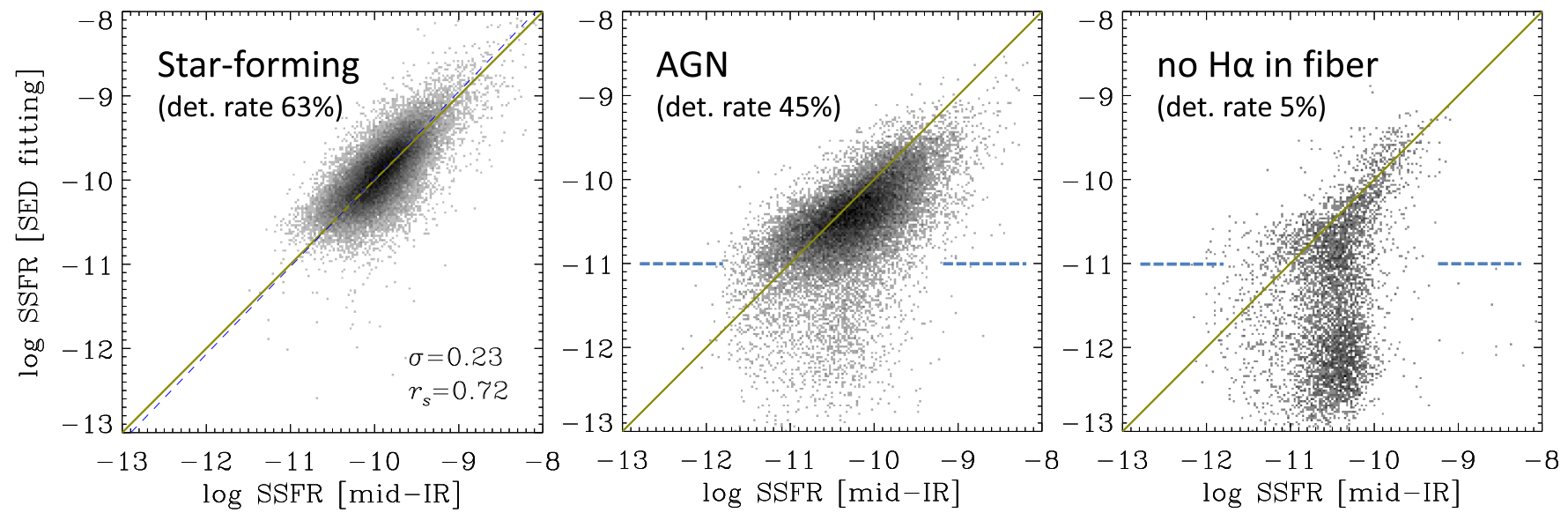

Figure 7. Comparison of UV/optical (SED fitting) and mid-IR sSFRs. Mid-IR sSFRs are derived from the $22 \mu \mathrm{m}$ mid-IR photometry from WISE (AllWISE catalog). Galaxies are split into SF and AGN classes according to the position in the BPT emission-line diagram. The AGN category includes all galaxies on the AGN branch (i.e., including the galaxies that B04 calls SF/AGN composites and LINERs). Galaxies with lines too weak to allow classification are presented separately in the right panel. The left panel plots SF galaxies and shows the best-fitting line along with its scatter and correlation coefficient. Galaxies below dashed lines in the middle and right panels have greatly overestimated mid-IR SFRs because their dust is primarily heated by old stars, invalidating the fixed $L_{\mathrm{IR}}-\mathrm{SFR}$ conversion. Their mid-IR SFRs have been removed from GSWLC. Both sSFRs use the stellar mass from our SED fitting. The detection rate at $22 \mu \mathrm{m}$ of a given class of galaxies is given in each panel. Data in this and subsequent figures are from GSWLC-M.

BPT lines and lying below the Kauffmann et al. (2003a) empirical demarcation between galaxies with and without an AGN contribution. As we have already seen in Section 5, once we have adopted an adequate attenuation law, the comparison is quite linear (slope in log-log plot equals 1), with no significant systematics. Mid-IR sSFRs from WISE are available for $63 \%$ of galaxies in this class. The scatter around the bisector linear fit (linear in $\log$ ) is $0.23 \mathrm{dex}$, to which the measurement errors of SED SFR and mid-IR SFR contribute approximately equally ( 0.15 dex each). Note that in all of the figures where we show the linear fit, we use a robust (outlier-resistant) bisector least-squares fit, which treats the variables symmetrically, i.e., no variable is considered independent.

Had we used mid-IR sSFRs based on unWISE photometry, the comparison would look similar, but it would have 5\% greater scatter. We find a similar level of degradation with respect to sSFRs based on $\mathrm{H} \alpha$. We have not positively identified the cause of the increased scatter of sSFRs based on unWISE compared to AllWISE photometry, but we list two possible reasons. unWISE uses $r$-band profiles to extract photometry. Optical light in $r$ band is dominated by the emission from older stellar populations, so it may not represent an optimal prior for $22 \mu \mathrm{m}$ observations, where the bulge emission is suppressed, while the star-forming regions in the disk dominate. Another possibility is related to the fact that unWISE is based on cmodelmag profiles, whereas all other SFRs are based on modelmag SDSS photometry. Despite being somewhat noisier in comparison with other (s)SFRs overall, unWISE IR luminosities and SFRs are less biased for large $\left(>10^{\prime \prime}\right)$ galaxies (where the AllWISE PSF magnitudes can underestimate the SFR by up to $0.1 \mathrm{dex}$ ), so we retain them in GSWLC alongside SFRs from the AllWISE catalog. Our recommendation is to use unWISE SFRs in studies that explore dependence on galaxy size or profile (e.g., Sérsic index) and also in studies that focus on galaxies with large angular diameter. Otherwise, AllWISE SFRs are recommended, and we continue to focus on them in subsequent discussion.

The middle panel shows the comparison for AGN-hosting galaxies. This category includes all galaxies above the Kauffmann et al. (2003a) line, i.e., what B04 call SF/AGN composites, AGNs (Seyferts), and LINERs (iclass = 3, 4, 5). AllWISE detects $45 \%$ of such galaxies. First, we notice that no correlation is present in the lower part of the plot, approximately when $\log \mathrm{sSFR}_{\mathrm{SED}}<-11$. These are nearly quiescent galaxies of LINER type (thus, potentially passive galaxies with non-AGN emission lines; Stasińska et al. 2008). For such galaxies the IR emission, if detected, will be dominated by dust heating by relatively dusty old or intermediate-age (e.g., AGB) stellar populations (Bressan et al. 2001; Villaume et al. 2015) and will therefore not be indicative of the current SF (Cortese et al. 2008; Salim et al. 2009). When the IR luminosity of such galaxies is converted into SFRs using simple, fixed-coefficient formulae (Equation (8)), which assume that dust is heated by young stars, the SFR will be overestimated (Buat \& Xu 1996; Kennicutt 1998; Boquien et al. 2016). For AGN galaxies with higher sSFRs, the mid-IR sSFRs tend to be up to 0.6 dex higher than the SED sSFRs, suggesting a non-negligible dust heating by AGNs, which produces excess emission in the mid-IR. The excess appears to be greater for increasing sSFRs, which would be expected if the gas both fuels the SF and drives the AGN accretion (e.g., Kewley et al. 2006).

Classification of a galaxy as a star-former or AGN, as performed by B04, requires minimum adjusted $\mathrm{S} / \mathrm{N}$ of 3 in $\mathrm{H} \alpha$ and other lines $(\approx 7.4$ in raw $\mathrm{H} \alpha \mathrm{S} / \mathrm{N})$. Galaxies that are too weak to allow classification, what we call the "no $\mathrm{H} \alpha$ " category (iclass $=-1$ ), are shown in the right panel. These galaxies are mostly quiescent, so it is not surprising that the AllWISE detection rate is only 5\%. When detected, the majority of galaxies in this class have low SED sSFRs, as expected. However, mid-IR sSFRs can be too high by up to 2 dex, because the IR emission from old stars is interpreted as current SF. We conclude that mid-IR SFRs are not reliable for quiescent or nearly quiescent galaxies when obtained through simple recipes that have a fixed conversion factor between IR luminosity and SFR (Equation (8)), and therefore in GSWLC we remove mid-IR SFRs for galaxies for which $\log \mathrm{SSFR}_{\mathrm{SED}}<-11$ (dashed line in middle and right panels of Figure 7). 

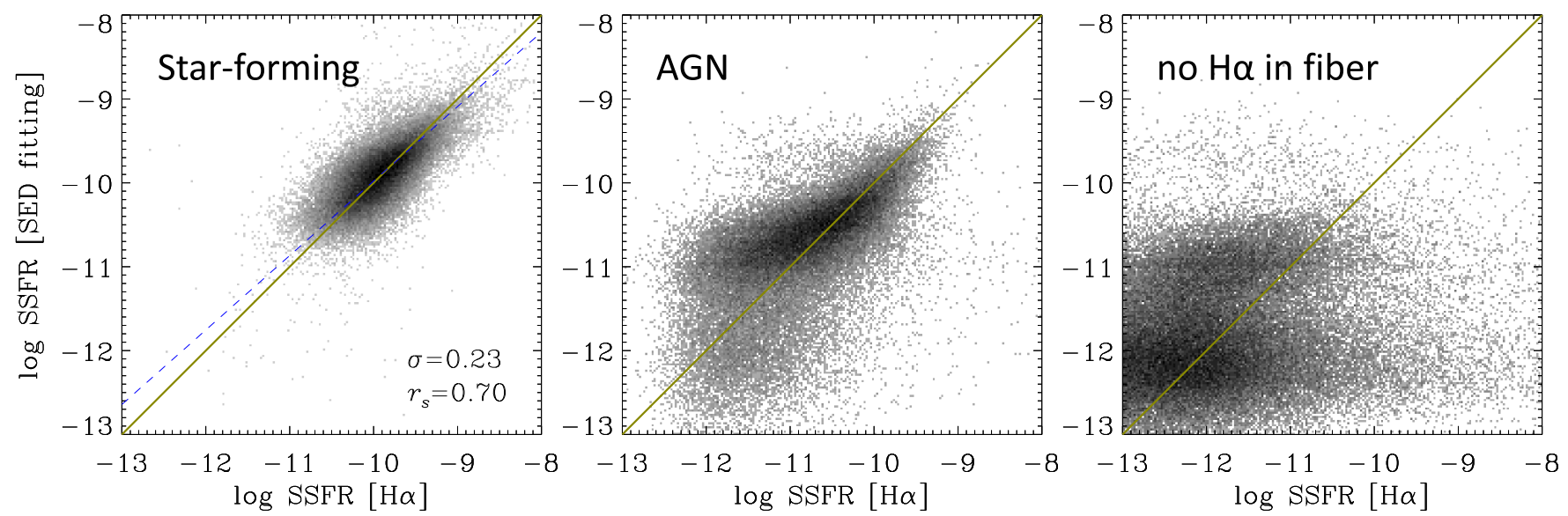

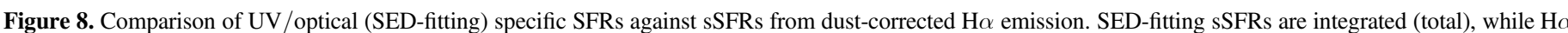

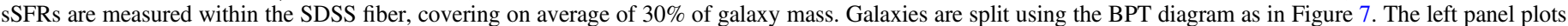
SF galaxies and shows the best-fitting line along with its scatter and correlation coefficient. H $\alpha$ sFR is normalized by stellar mass within the fiber.

Interestingly, there are some galaxies in the "no $\mathrm{H} \alpha$ " class for which the SED-fitting sSFRs are quite high, overlapping with the values of normal star-forming galaxies (log sSFR $>-10.5$ ). It may seem contradictory that a galaxy with essentially no $\mathrm{H} \alpha$ emission could have such high sSFR. There are two possible reasons for this: (1) The difference in SF timescales. $\mathrm{H} \alpha$ is not present because a galaxy is in a poststarburst phase-UV and mid-IR emission will persist after the $\mathrm{O}$ stars that give rise to $\mathrm{H} \alpha$ emission have died off. In other words, high SED and mid-IR sSFRs reflect recent, but not instantaneous, values. The post-starburst galaxies would form the tail of high sSFRs in Figure 7 (right; $\log$ sSFR $>-10$ ). Their SED and mid-IR sSFRs agree, with no mid-IR excess like the one seen for AGNs. This suggests that post-starburst galaxies, which are usually considered to be the results of mergers (e.g., Yang et al. 2008), nonetheless do not have a significant AGN emission. (2) $\mathrm{H} \alpha$ is not present within the SDSS fiber, but the SF (and presumably the $\mathrm{H} \alpha$ emission) is present outside of it. Salim et al. (2012) and Fang et al. (2012) have studied such a population in detail with high-resolution $\mathrm{UV}$, optical, and $\mathrm{H} \alpha$ imaging and have confirmed that these are typically lenticular (S0) galaxies with no SF in the bulge, but with low-level SF present in a ring outside of the SDSS fiber. The sSFRs of such star-forming S0s would be in the range $-12<\log$ sSFR $<-10$, i.e., lower than post-starburst galaxies.

Figure 8 shows an equivalent comparison of SED sSFRs with respect to $\mathrm{H} \alpha$ sSFRs. $\mathrm{H} \alpha$ sSFRs are measured within the 3 " SDSS fiber, which covers between $17 \%$ and $50 \%$ of the galaxy's stellar mass. The comparison of star-forming galaxies (left panel) shows similar scatter to that seen with respect to the mid-IR. The relation is somewhat sSFR dependent. One expects the relation between total and fiber sSFR to be linear (slope in log-log plot equal to 1) only if there are no sSFR gradients. Somewhat increased nonlinearity for galaxies with $\log \mathrm{sSFR}_{\mathrm{H} \alpha} \geqslant-9$ suggests either that the SF tends to be centrally concentrated, so that it produces higher sSFRs within the fiber compared to the integrated SSFR, or that the fiber mass from the MPA/JHU catalog, which we use to normalize the $\mathrm{H} \alpha$ SFR, is systematically underestimated for bursty galaxies (Section 8.1).

For galaxies with an AGN/LINER contribution (middle panel), there are no systematic differences for hosts having high
SF (log sSFR $>-10)$. This is in contrast to mid-IR sSFRs that showed an excess in this regime. $\mathrm{H} \alpha$ sSFRs start to display systematic discrepancies at lower sSFRs, in the sense that $\mathrm{H} \alpha$ sSFRs are up to 0.7 dex too low. We find that many such cases are galaxies with no optical signs of SF, either photometrically (optical colors are red) or spectroscopically (not much $\mathrm{H} \alpha$ ), and yet the UV is clearly indicative of SF-often the UV colors are noticeably blue and uniformly spread across the disk. These galaxies may be in a declining phase of SF where the UV emission is higher than the rapidly diminishing $\mathrm{H} \alpha$.

For galaxies with "no $\mathrm{H} \alpha$ " (right panel), no correlation is present, as expected given the weakness of $\mathrm{H} \alpha$ that defines this category and making $\mathrm{H} \alpha$ sSFRs essentially meaningless. There is an overdensity of galaxies below $\log \mathrm{SSFR}_{\mathrm{SED}}=-12$, where SED SFR estimates also become rather uncertain (Figure 6) and are more likely just the upper limits of galaxies with no current SF whatsoever, such as the majority of earlytype galaxies.

To conclude, for actively star-forming galaxies all three indicators (SED fitting, mid-IR, and $\mathrm{H} \alpha$ ) provide robust measurements of (s)SFR. For mid-IR, the depth of WISE allows only two-thirds of SDSS galaxies to be detected. $\mathrm{H} \alpha$ (s) SFRs pertain only to the fiber. Mid-IR luminosity breaks down as an SFR indicator for galaxies with $\log \mathrm{SSFR}_{\mathrm{SED}}<-11$, where dust heating is dominated by old populations. This corresponds to green-valley and quiescent galaxies. Furthermore, the mid-IR SFR appears to be affected by AGN emission, limiting its usefulness. SED sSFRs are measurements of choice in the low-sSFR regime, but even they become essentially upper limits when $\log \mathrm{SSFR}_{\mathrm{SED}}<-11.7$ (for GSWLC-M; -12.0 for GSWLC-D; -11.5 for GSWLC-A).

\section{COMPARISON OF GSWLC WITH PREVIOUS CATALOGS}

In this section we compare GSWLC stellar masses and SEDfitting SFRs (primarily through sSFRs) with those from previously published catalogs. The focus will be on (s)SFR comparisons, which are more sensitive than stellar mass measurements. We continue to present the comparisons using GSWLC-M, but the results hold with GSWLC-A and GSWLC-D. 


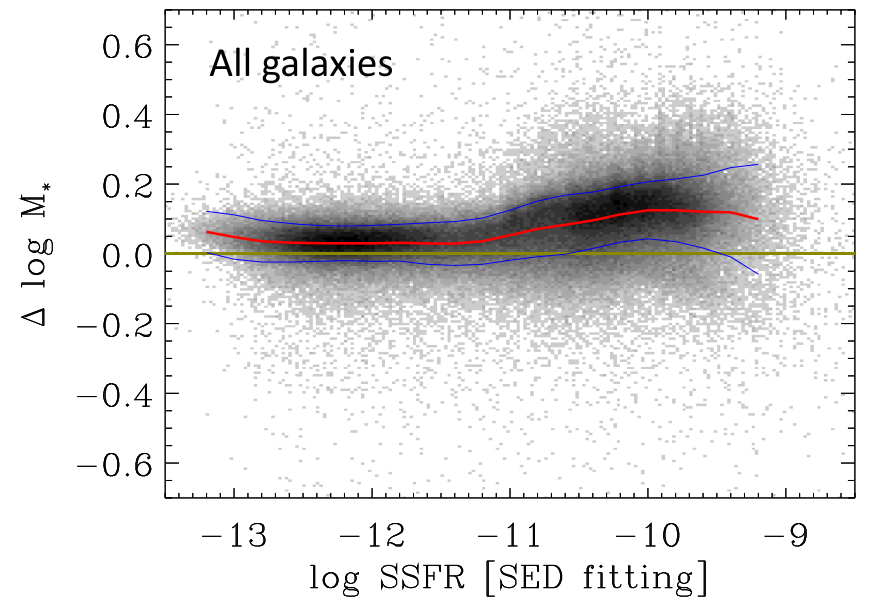

Figure 9. Comparison of stellar masses from GSWLC-M and MPA/JHU (DR7) catalogs, presented as the difference in masses as a function of sSFR. The red curve gives the averages of the difference in 0.2 dex wide bins, and the blue curves are the averages $\pm 1 \sigma$. GSWLC masses are on average higher than MPA/JHU catalog masses because of the differences in the assumed SF histories, but the difference is quite small (0.03-0.13 dex) and is sSFR dependent. The standard deviation of the mass difference is typically 0.07 dex.

\subsection{Comparison with MPA/JHU Stellar Masses}

The MPA/JHU catalog is the most widely used source of stellar masses for SDSS galaxies. Prior to DR7, the most recent version of this catalog, the MPA/JHU catalog listed stellar masses derived in Kauffmann et al. (2003b), using a method that combines photometric information to assess dust attenuation and spectroscopic indices to constrain the SF history and mass-to-light ratio. Spectroscopic indices were measured within the fiber, so the method assumed no $M / L$ gradients. In the DR7 version of the MPA/JHU catalog, the method from Kauffmann et al. was replaced with the Bayesian SED-fitting method, using only the optical broadband photometry from SDSS (modelMag) and models described in S07. Here we will only present comparisons with respect to the DR7 version of the MPA/JHU catalog.

As described in Section 5, GSWLC uses different specifications of SF histories and dust extinction from those used in S07 and the MPA/JHU catalog. Furthermore, unlike the MPA/ JHU catalog (but like S07), GSWLC uses the constraints offered by UV photometry. Figure 9 shows the difference between GSWLC and MPA/JHU stellar masses as a function of sSFR. Again, we prefer the presentation of the results in this relative, distance-independent way over the usual mass versus mass comparison. On average, GSWLC stellar masses are somewhat higher than the MPA/JHU ones, with typical differences being 0.03 dex for passive galaxies and $\sim 0.13$ dex for the active ones. The scatter of the difference in mass, $0.07 \mathrm{dex}$, is consistent with the formal estimates of the mass error (Figure 6). The inclusion of the UV photometry is responsible for a 0.04 dex increase for active galaxies. The remaining 0.09 dex increase for active galaxies (and 0.03 dex increase for passive ones) is mostly due to our use of twocomponent exponential SF histories. We confirm that the difference would not have been present if we had used the delayed exponential histories instead (Equation (9)). The critical difference between the delayed exponential and our implementation of the two-component exponential SF history is that in the latter the old component starts in the early universe, whereas the delayed exponential, having a single peak, will be shifted toward later epochs. Recently, Sorba \& Sawicki (2015) have reported that the masses of nearby highsSFR galaxies obtained by summing up the masses in individual "pixels" are up to $0.1 \mathrm{dex}$ higher than the masses from integrated light. They proposed that the difference arose from "outshining" of the old, fainter populations in galaxies with younger populations (high sSFR). It appears that our use of the two-component exponential SF history, in which the old component is set to have started in the early universe, may have recovered this deficit.

More recently, stellar masses for SDSS galaxies were published by Mendel et al. (2014) and Chang et al. (2015). The differences of GSWLC masses with respect to these masses follow the trends we have shown with respect to masses from the MPA/JHU catalog: little difference for passive galaxies, and up to 0.2 dex difference for galaxies with high sSFR.

Comparison of our masses obtained with and without the UV photometry reveals that in the case when there is another photometric source within $2^{\prime \prime}-3^{\prime \prime}$, the masses obtained from joint UV and optical photometry will be biased upward by 0.05 dex, presumably because of the unaccounted blending in the UV. This affects only a few percent of all sources.

\subsection{Comparison with MPA/JHU SFRs}

The MPA/JHU catalog also provides SFRs, and until recently it was the only publicly available source of SFRs for SDSS galaxies. SFRs derived in the MPA/JHU catalog follow the method of B04, with modifications introduced in the most recent (DR7) version of the catalog. We first describe both the original method and its modifications, but the comparison will be presented only for the DR7 version of the MPA/JHU catalog.

MPA/JHU catalog (and the original B04) SFRs are often described as $\mathrm{H} \alpha$ or emission-line SFRs. This is accurate only for the portion of a galaxy contained within the fiber, and only for galaxies classified as star-forming using the BPT diagram, for which the AGN contribution to emission lines should be negligible. For galaxies with an AGN contribution or having weak lines (altogether $78 \%$ of SDSS galaxies), B04 derive SFR in the fiber based on a relation between the emission-line sSFR and D4000 index, constructed from star-forming galaxies. Next, the SFR estimate within the fiber is aperture-corrected to produce the total (integrated) SFR. B04 performs this correction by first establishing the relationship between fiber sSFR and the fiber broadband colors of star-forming galaxies and then applying these relations in a Bayesian fashion to the light outside of the fibers to arrive at the out-of-fiber SFR. The total SFR is then obtained as the sum of fiber and out-of-fiber SFRs. The DR7 MPA/JHU catalog modifies the procedure for deriving out-of-fiber SFRs, by instead performing the SED fitting to ugriz photometry, using the models and methods described in S07. Altogether, the SFR method of the MPA/ JHU catalog (and original B04) is an emisison-line/D4000/ SED-fitting hybrid. The temporal sensitivity of such SFRs will be between the $\sim 10 \mathrm{Myr}$ timescales traced by the emission lines and the $\sim 1$ Gyr timescale for $u$-band light.

S07 presented detailed comparison of their SED SFRs and the original SFRs from B04. GSWLC contains many improvements over the S07 methodology, as does the MPA/ JHU catalog with respect to B04. The comparison of GSWLC$\mathrm{M}$ and DR7 MPA/JHU total sSFRs is given in Figure 10. It 

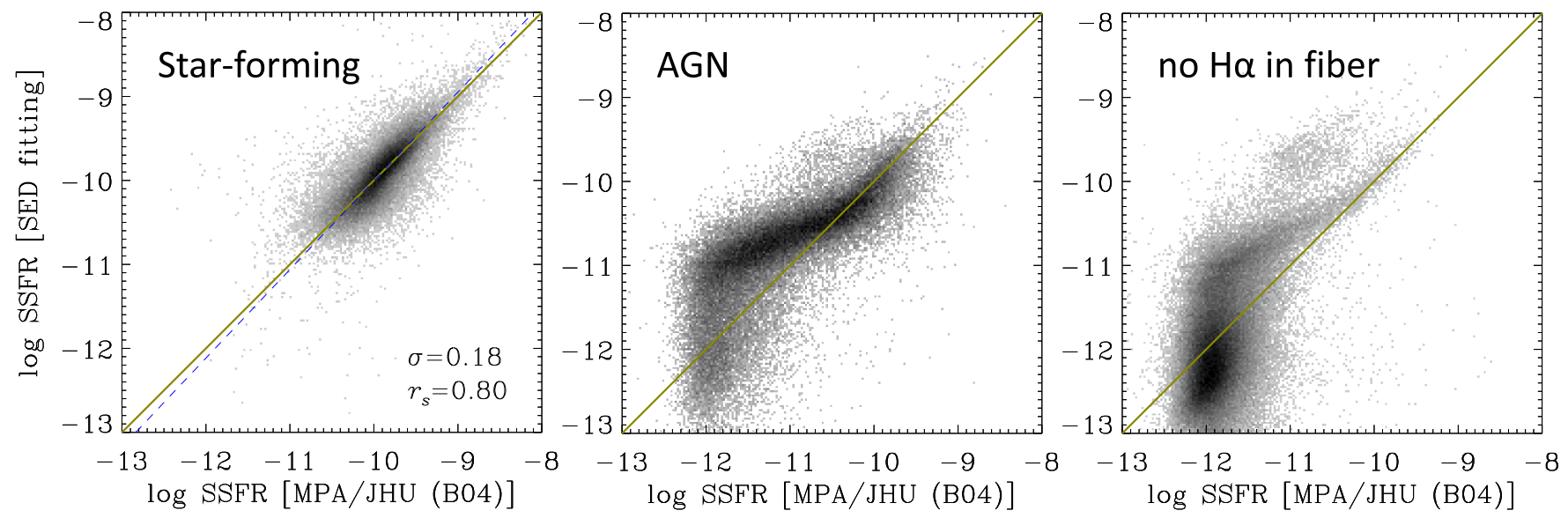

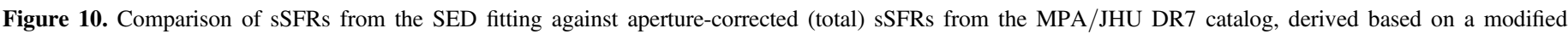

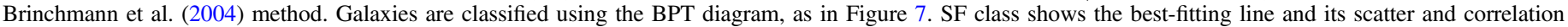

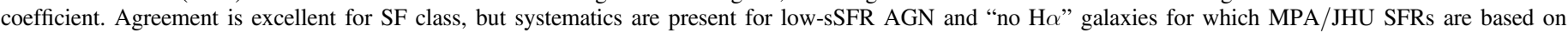
indirect methods that are not sensitive to low levels of SF.

uses the same division of galaxies into $\mathrm{SF}, \mathrm{AGN}$, and "no $\mathrm{H} \alpha$ " categories as employed earlier. In order to decouple SFR and mass systematics, both sSFRs are obtained by normalizing by the same mass (from GSWLC). Note that for 3\% of galaxies in the MPA/JHU catalog the total SFRs are present even when masses are not. We find that in those cases the reported total SFR is incorrect and assumes the value of fiber SFR. We exclude those values from the comparison.

sSFRs for star-forming galaxies (left panel) agree very well, with a scatter of $\sigma=0.18$, which is smaller than the scatter of SED sSFRs with respect to mid-IR or $\mathrm{H} \alpha$ sSFRs (Figures 7 and 8). The comparison for AGN hosts reveals some systematic discrepancies, especially for intermediate sSFRs, which can in some cases reach $\sim 1$ dex. We have already seen similar, but smaller, offsets in comparison with $\mathrm{H} \alpha$ fiber sSFRs, which we attributed to optically (spectroscopically and photometrically) inconspicuous SF. If we intentionally leave out the UV bands from our SED fitting, the resulting (ill-constrained) sSFRs are drawn to lower values (because the majority of models with red optical colors have low sSFRs), in better agreement with B04 values, which derive the greater part of their SFR (the out-offiber portion) from similar optical-only SED fitting.

Similar trends are present in galaxies with little or no $\mathrm{H} \alpha$ emission in the fiber (right panel), except that the majority of galaxies have low sSFRs $\left(\log \mathrm{sSFR}_{\mathrm{SED}}<-11\right)$. In this class of galaxies there exists a peculiar feature: a cloud of galaxies lying $\sim 1$ dex above the 1:1 line, having high SED sSFRs $\left(\log \mathrm{sSFR}_{\mathrm{SED}}>-10\right)$, but much lower MPA/JHU sSFRs. We confirm that these galaxies have robust SED fits and are UV detected. In Section 7 we mentioned that "no $\mathrm{H} \alpha$ " galaxies with high sSFRs are probably post-starburst galaxies. Such galaxies are traditionally identified as E+A galaxies, based on their Balmer absorption features (Dressler \& Gunn 1983). Now we inspect the spectra of $\sim 200$ of these outliers and confirm the presence of Balmer absorption lines. Visually (gri composites), these galaxies appear like red early-type galaxies, often with white centers, suggesting a central (post-)starburst. We match our sample to the Goto (2007) catalog of $\mathrm{E}+\mathrm{A}$ galaxies (online version updated with SDSS DR7 data) and confirm that the majority of their E+As are found in this region of the plot. Why do the two methods yield discrepant SFRs for these galaxies? We find that the SED-fitting (s)SFRs of E+A galaxies are very sensitive to the assumed dust attenuation law, more so than the normal star-forming galaxies. The (s)SFRs that we obtain for E+As assuming the modified attenuation law are an order of magnitude higher than what would be derived using the nominal Calzetti law. The reduced $\chi^{2}$ s are 5 times lower when the modified attenuation law is used. From this we conclude that the high recent (s)SFRs of $\mathrm{E}+\mathrm{As}$ that we derive in GSWLC are more likely to represent true levels of SF averaged over the past $100 \mathrm{Myr}$.

\subsubsection{On the Systematics of MPA/JHU SFRs Reported by SAMI Galaxy Survey}

Recent campaigns to obtain resolved spectra with integral field spectroscopy have reported systematic differences with respect to SFRs from the MPA/JHU catalog. In particular, Richards et al. (2016), using preliminary data from the SAMI Galaxy Survey (Allen et al. 2015), find a nonlinear, i.e., SFRdependent, relation between their and MPA/JHU SFRs (which they refer to as B04 SFRs), in the sense that galaxies with high SAMI SFRs have underestimated MPA/JHU SFRs. The discrepancy is already $\sim 0.3 \mathrm{dex}$ at $\log \mathrm{SFR}_{\mathrm{SAMI}} \approx 0.6$, the highest SFRs in their sample (we convert all SFRs from Richards et al. to Chabrier IMF). Richards et al. attribute the discrepancy to possible biases in B04 methodology for deriving aperture corrections.

In our analysis so far we have shown that GSWLC specific SFRs of star-forming galaxies have no significant systematics with respect to either mid-IR sSFRs or sSFRs from the MPA/ JHU catalog. This implies, and we confirm it to be true, that MPA/JHU and mid-IR sSFRs agree between themselves as well. While this seems to imply that MPA/JHU measurements are not biased, it is necessary to verify if such results hold for SFRs, and not just the sSFRs.

Figure 11 shows SED-fitting SFRs against MPA/JHU SFRs in the upper panel and mid-IR SFRs against MPA/JHU SFRs in the lower panel. In both comparisons, the linear fits (blue dashed lines) follow closely the 1:1 relation. White solid lines show the relation between SAMI and B04 SFRs (adjusted to Chabrier IMF), over the range of SFRs covered in Richards 

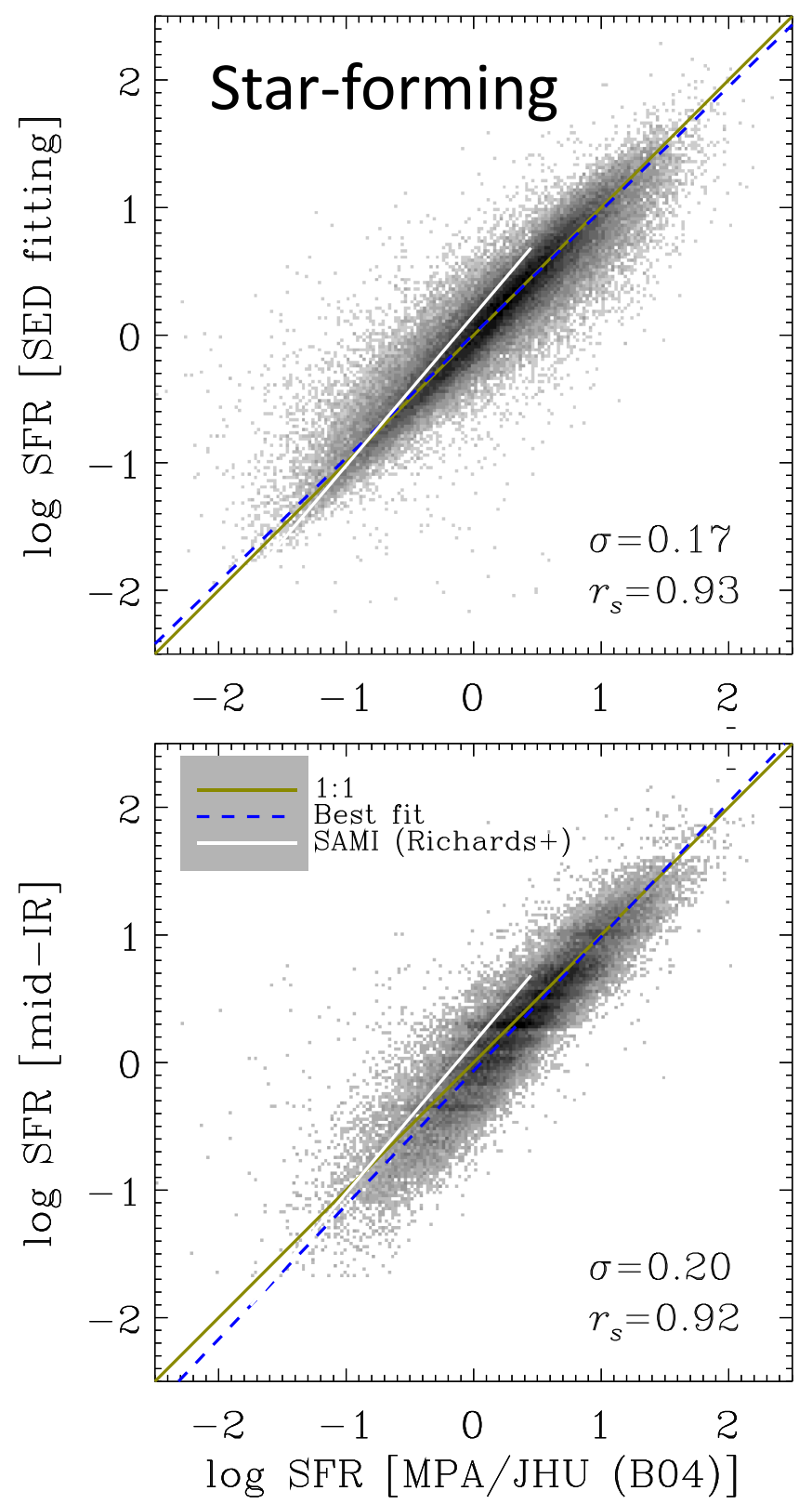

Figure 11. Comparison of SFRs from the SED fitting (upper panel) and from WISE mid-IR (lower panel), with respect to SFRs from the MPA/JHU catalog (Brinchmann et al. 2004). Shown are the galaxies from GSWLC-M classified as star-forming on the BPT diagram. There are no systematic differences or significant nonlinearities. This is in contrast with the relation derived from integral field spectroscopy $\mathrm{H} \alpha$ measurements from the SAMI Galaxy Survey (Richards et al. 2016), shown as the white solid line (SAMI vs. MPA/JHU). In this figure we lower the redshift limit to $z_{\min }=0.01$ to increase the contribution of low-SFR galaxies. Slight discretization in mid-IR SFRs arises from sampling of Chary \& Elbaz (2001) IR templates.

et al. (2016). ${ }^{17}$ The discrepancies suggested by the Richards et al. relation are clearly excluded with either the SED-fitting or the mid-IR SFR. Results are unchanged when the redshift range of galaxies is restricted to match the redshift range of the SAMI Galaxy Survey. Apparently, more work is needed to understand the source of differences. For now, we conclude that the total MPA/JHU SFRs of actively star-forming galaxies do not

\footnotetext{
17 The highest SFRs in Richards et al. (2016) are an order of magnitude lower than the highest SFRs in SDSS, probably because their sample is drawn from a much smaller volume compared to that of SDSS (smaller area, plus $z<0.06$ ).
}

appear to be biased when compared with two independent measures of integrated SFR.

\subsection{Comparison with Ellison et al. (2016) SFRs}

All-sky far-IR observations that would enable direct measurement of the total IR luminosity across SDSS are only available from relatively shallow IRAS and AKARI surveys, with preferentially more luminous galaxies (Ellison et al. 2016). In order to produce IR luminosity estimates for a significant portion of the SDSS spectroscopic sample, Ellison et al. (2016) apply an artificial neural network (ANN) technique, using IR luminosities from Rosario et al. (2016) as the training set (these IR luminosities were discussed in Section 4). The idea behind the ANN is to establish intrinsic correlations between observable quantities of the training set and the target quantity (in this case the IR luminosity) and then apply these correlations to estimate ("predict") the target quantity in the full data set. The robustness of ANN estimates should be tested by comparing them with the independent measurements of the target quantity for an unbiased subsample drawn from the full data set. In the case of Ellison et al. (2016), the parameters employed to estimate the IR luminosities using ANN include, in addition to the redshift, the photometric (magnitudes, colors) and spectroscopic measurements (emission-lines strengths and D4000 break), as well as the stellar mass. The connection between the fiber and total quantities is established by also including the mass in the fiber and the $r$ band fiber covering fraction. The input parameters come from the MPA/JHU DR7 catalog. The requirement to have available all of the parameters in the target data set limits the application to $45 \%$ of SDSS galaxies, typically the ones with stronger emission lines. IR luminosities of the training set are recovered with the typical accuracy of $\sigma \sim 0.1 \mathrm{dex}$, with no major systematics (Ellison et al. 2016).

In Figure 12 we show the comparison of GSWLC sSFRs from the SED fitting against sSFRs converted from Ellison et al. (2016) IR luminosities using Equation (8). The comparison is limited to galaxies with $\sigma_{\mathrm{ANN}}<0.1$, a cut recommended in Ellison et al. (2016) to remove the galaxies whose estimated IR luminosities may be uncertain. $\sigma_{\mathrm{ANN}}$ essentially measures the degree to which a target galaxy is represented in the training set. The larger the value, the less likely the target galaxy is represented in the training set, and its IR luminosity may therefore be uncertain. The application of the cut on $\sigma_{\mathrm{ANN}}$ decreases the number of galaxies with ANN IR luminosities from 330,000 to 250,000 , or one-third of SDSS. The comparison of sSFRs of star-forming galaxies (left panel) shows good general agreement, with mild nonlinearity (slope of 1.23), which can be traced to the differences between WISE and Herschel-WISE specific IR luminosities (Section 4). If the galaxies with $\sigma_{\mathrm{ANN}}>0.1$ were included in the comparison, the scatter at high sSFRs would increase, presumably because such galaxies are rare in the training set. ANN IR luminosities are available for $77 \%$ of galaxies in this class (after the application of the $\sigma_{\mathrm{ANN}}$ cut), which is higher than the WISE detection rate of $63 \%$.

For galaxies classified as AGNs (the middle panel), the correlation is present when $\log \mathrm{sSFR}_{\mathrm{SED}}>-11$, but with ANN sSFRs tending to be higher, especially for galaxies with high sSFR. Below log sSFR ${ }_{\mathrm{SED}}=-11$ there is no correlation. Both of these behaviors mimic the comparison of SED-fitting sSFRs with mid-IR sSFRs (Section 7 and Figure 7, middle panel). 

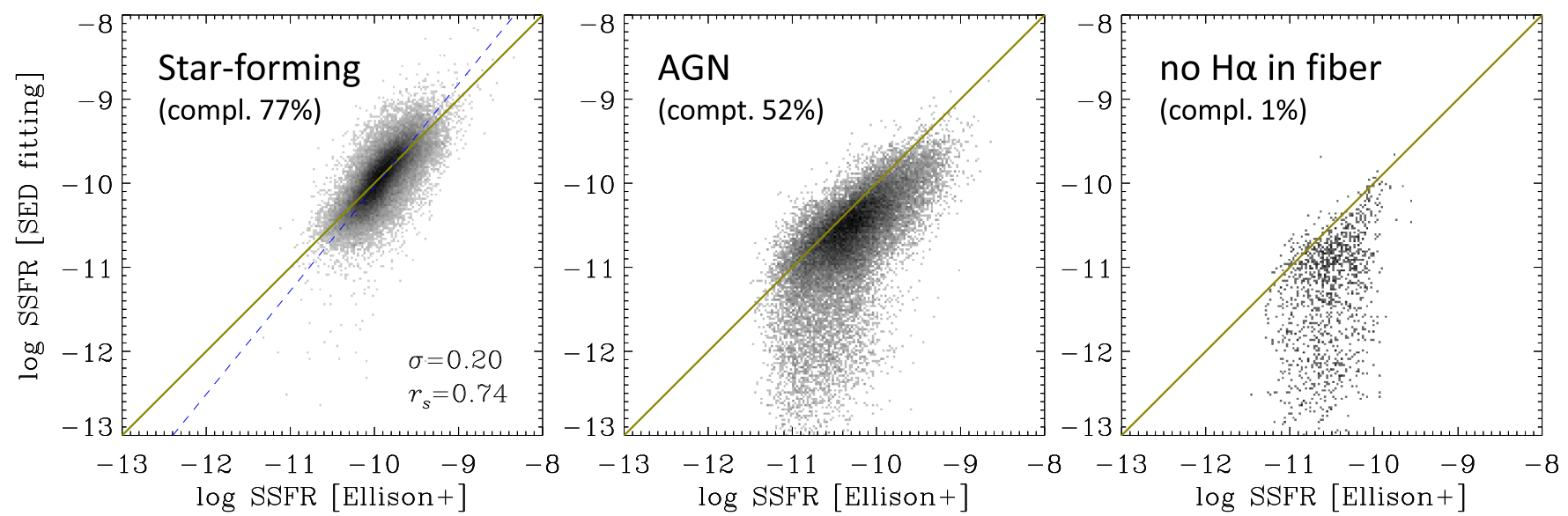

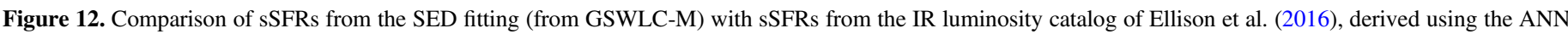

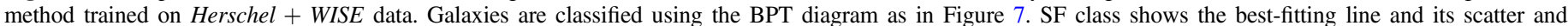

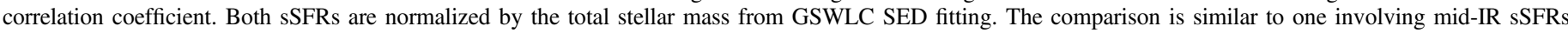

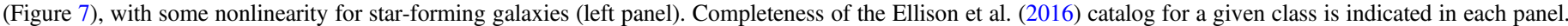

Excess sSFR for AGNs with higher sSFRs, which in the case of mid-IR sSFRs we attributed to AGN dust heating, is somewhat surprising, because it would be expected that the AGN contribution, which drops above $40 \mu \mathrm{m}$ (Mullaney et al. 2011), will be significantly diminished in the total IR luminosity. However, we remind the reader that ANN luminosities are trained on IR luminosities derived from a combination of $22 \mu \mathrm{m}$ flux (which is subject to AGN contamination) and three submillimeter flux points that lie well beyond the IR SED peak (and are therefore not as sensitive to the current $\mathrm{SF}$, and more to the cold dust mass). More detailed analysis on the contribution of type 2 AGNs to IR SEDs lies outside of the scope of this paper.

After the application of the $\sigma_{\mathrm{ANN}}$ cut, the IR luminosities from Ellison et al. (2016) are available for only $1 \%$ of galaxies having weak or no $\mathrm{H} \alpha$ (right panel). For a handful of such objects with high sSFRs (including confirmed E+As) the match between the SED-fitting and ANN sSFRs is good, but for the great majority, the ANN IR luminosities, when interpreted as SFRs, tend to be too high because of the dust heating from older stars, as already discussed in Section 7.

Considering the very different methods of estimating the IR luminosities, it is interesting to see how the ANN IR luminosities from Ellison et al. (2016) compare to the ones we obtain from WISE. This is shown in Figure 13, for all galaxies for which the two measurements are available (and with the $\sigma_{\mathrm{ANN}}<0.1$ cut applied). The comparison, extending over three orders of magnitude, is fairly good, in terms of both offset and the scatter $(\sigma=0.17)$. Some systematic differences are present, especially at $\log L_{\mathrm{IR}}>11$, which is not surprising considering that the training set peters out at those luminosities. Overall, the IR luminosities from Ellison et al. (2016) represent remarkably good estimates considering that they were determined from optical properties alone, but their use as SFRs is subject to the same caveats (AGN dust heating and breakdown for passive galaxies) as the SFR obtained from WISE mid-IR data.

\subsection{Comparison with Chang et al. (2015) SFRs}

In Section 4 we mention that new SED-fitting codes allow the modeling of the SED to extend into the IR, by including the dust emission. This approach is used in MAGPHYS (da Cunha

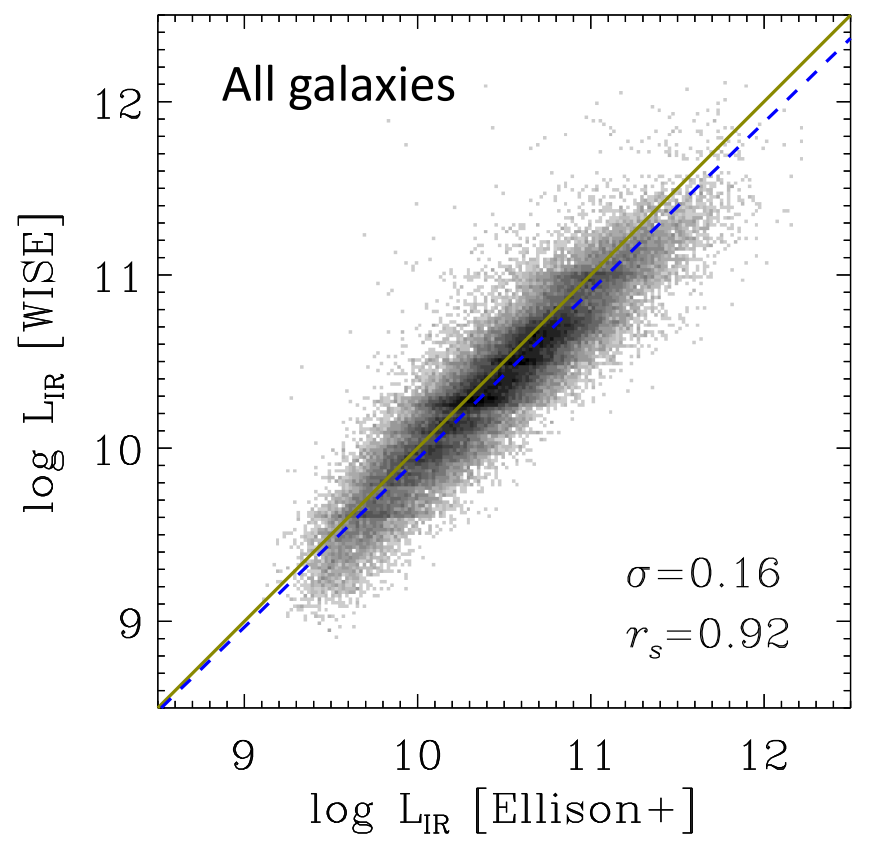

Figure 13. Comparison of total IR luminosities derived in GSWLC using WISE against the total IR luminosities derived with ANN in Ellison et al. (2016). WISE measurement is direct, but is based on extrapolating a single flux point at rest frame $\sim 20 \mu \mathrm{m}$. ANN, on the other hand, uses various optical photometric and spectroscopic measurements to predict the IR luminosity based on a training set consisting of IR luminosities from Herschel + WISE (three submillimeter flux points from SPIRE and $22 \mu \mathrm{m}$ points from WISE). The comparison is good, notwithstanding some systematics for the most luminous galaxies. Luminosities are given in solar units.

et al. 2008), which models the IR SED as a sum of various SED components, the relative contribution of which is mildly related to the galaxy's sSFR. CIGALE allows the IR SED to be modeled according to one of the four published template sets, without constraints on the shape. In both cases the dust luminosity (i.e., the total IR luminosity) is normalized to match the stellar emission absorbed in the UV/optical/near-IR. Such IR luminosity will therefore include dust heating from stars of all ages.

Stellar plus dust emission modeling is applied in Chang et al. (2015), who use MAGPHYS to perform the SED fitting simultaneously on optical (ugriz) photometry from SDSS and 

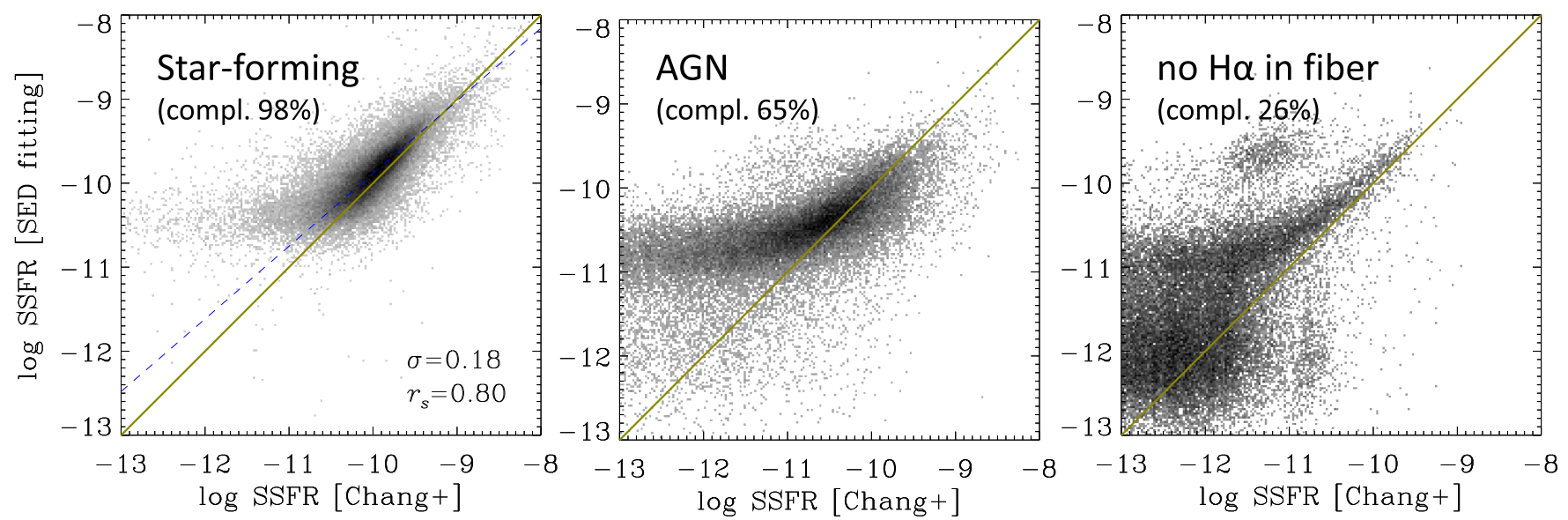

Figure 14. Comparison of sSFRs from the SED fitting against the sSFRs from Chang et al. (2015), derived from optical+mid-IR SED fitting using MAGPHYS (da Cunha et al. 2008). Galaxies are classified using the BPT diagram as in Figure 7. SF class shows the best-fitting line and its scatter and correlation coefficient. Both sSFRs are normalized by the total stellar mass from GSWLC SED fitting. Low sSFRs tend to be underestimated in the Chang et al. (2015) catalog, but otherwise the correlation is good with a small scatter. Completeness of the Chang et al. (2015) catalog for a given class is indicated in each panel.

mid-IR photometry from $\operatorname{WISE}(3.4,4.6,12$, and $22 \mu \mathrm{m})$. Of these nine bands, all but the longest two WISE bands will be dominated by the stellar emission. Emission at 12 and $22 \mu \mathrm{m}$, if detected, will help constrain the dust luminosity and therefore the SFR (if the dust is primarily heated by young stars).

We follow the analysis established in previous sections, and in Figure 14 we present comparisons between our SED-fitting sSFRs and sSFRs from Chang et al. (2015), again split by galaxy type. ${ }^{18}$ Note that we normalize both sSFRs using our stellar mass. Galaxies classified as star-forming on the BPT diagram (left panel) compare well, but show a tail of anomalously low values of Chang et al. sSFRs, which causes the best-fit line to deviate from unity. However, if the tail is excluded, the nonlinearity disappears, and a small bulk offset of 0.09 dex remains. We find that the offset is entirely due to the $\sim 1 / 3$ of galaxies that are not detected at $22 \mu \mathrm{m}$ but only at $12 \mu \mathrm{m}$, for which Chang et al. (s)SFRs are on average $0.17 \mathrm{dex}$ lower than our SED-fitting (s)SFRs. For galaxies with $22 \mu \mathrm{m}$ detection, the scatter with respect to SED sSFRs is 0.17 dex, compared to 0.23 dex between SED sSFRs and sSFRs from WISE $22 \mu \mathrm{m}$ alone (Figure 7, left panel). This reduction of scatter demonstrates that the $12 \mu \mathrm{m}$ photometry (a wavelength at which WISE is significantly more sensitive than to $22 \mu \mathrm{m}$ ) helps constrain the SFRs compared to when $22 \mu \mathrm{m}$ is used alone. However, when $12 \mu \mathrm{m}$ is used to obtain the IR luminosity (or SFR) without the $22 \mu \mathrm{m}$ measurements, it leads to a systematic underestimate.

For AGN hosts (the middle panel), the tail of anomalously low sSFR values is more extensive than for the star-forming galaxies. We confirm that these galaxies have UV detections and that the UV emission drives the sSFR estimate in our SED fitting to higher values than when the UV is omitted from the SED fitting, as in the case of the Chang et al. (2015) SED fitting. UV is not expected to be contaminated by nonstellar emission in these (type 2) AGNs. Furthermore, the UV emission is extended, suggestive of SF. Optical colors alone are not sensitive to such low levels of SF (Kauffmann et al. 2007) and are equally red for truly quiescent galaxies and those with intermediate sSFRs (Fang et al. 2012). When the SED

\footnotetext{
${ }^{18}$ We find that restricting the comparison to galaxies that Chang et al. (2015) flag as having good determinations has negligible effect on the results, so we use all galaxies regardless of that flag.
}

fitting is performed without UV constraints, the sSFR defaults to very low values of the majority of optically red models.

For AGN hosts with high sSFRs, the excess seen in other IRbased sSFRs is now smaller. This is true whether the galaxy was detected at both 12 and $22 \mu \mathrm{m}$ or just at $12 \mu \mathrm{m}$, suggesting that $12 \mu \mathrm{m}$ flux is much less affected by AGN-heated hot dust than the $22 \mu \mathrm{m}$, so that when included in the SFR estimate it mitigates the excess. That $22 \mu \mathrm{m}$ suffers more AGN contamination is corroborated by the fact that out of $12 \mu \mathrm{m}$-detected AGNs, $80 \%$ are also detected at $22 \mu \mathrm{m}$, whereas this fraction was $60 \%$ for star-forming galaxies. Previously, Donoso et al. (2012) have found that the $12 \mu \mathrm{m}$ luminosity is not affected by an AGN contribution in all but a handful of the most luminous sources (but they did not perform analogous assessment of $22 \mu \mathrm{m}$ emission).

For galaxies with weak or no $\mathrm{H} \alpha$ (right panel), the Chang et al. (2015) values agree well for some of the galaxies with high sSFRs, with a small offset that we attribute to the preponderance $(90 \%)$ of $12 \mu \mathrm{m}$-only detections in this category, which we have shown to have somewhat underestimated SFRs in Chang et al. As in the case of comparison with MPA/JHU catalog SFRs, the E+As, or more generally, the post-starburst galaxies, again form a cloud of points offset from the 1:1 relation. As discussed in Section 8.2, the offset in (s)SFRs is attributable to the differences in the assumed dust attenuation laws, to which the E+As appear to be particularly sensitive. Comparison with entirely independent IR SFRs, as well as better quality of SED fits, suggests that the high (s)SFRs obtained with the modified attenuation law in GSWLC are more realistic. Finally, for galaxies in the "no $\mathrm{H} \alpha$ " class with $\log$ sSFR $<-11$ the estimates largely agree, but are quite uncertain.

The general conclusion is that the Chang et al. (2015) SFRs are reliable for galaxies with $\log \mathrm{sSFR}_{\mathrm{C} 15}>-10.7$, regardless of the galaxy type, and especially when a galaxy is detected at $22 \mu \mathrm{m}$. When sSFRs are low, Chang et al. (2015) correctly attribute the mid-IR emission to old populations and not the ongoing SF. However, in doing so, the weak signal from the actual SF, detectable in the UV, is in some cases lost, leading to anomalously low values of sSFR.

Chang et al. (2015) provide an estimate of the dust luminosity, i.e., the total IR luminosity. In Figure 15, we 


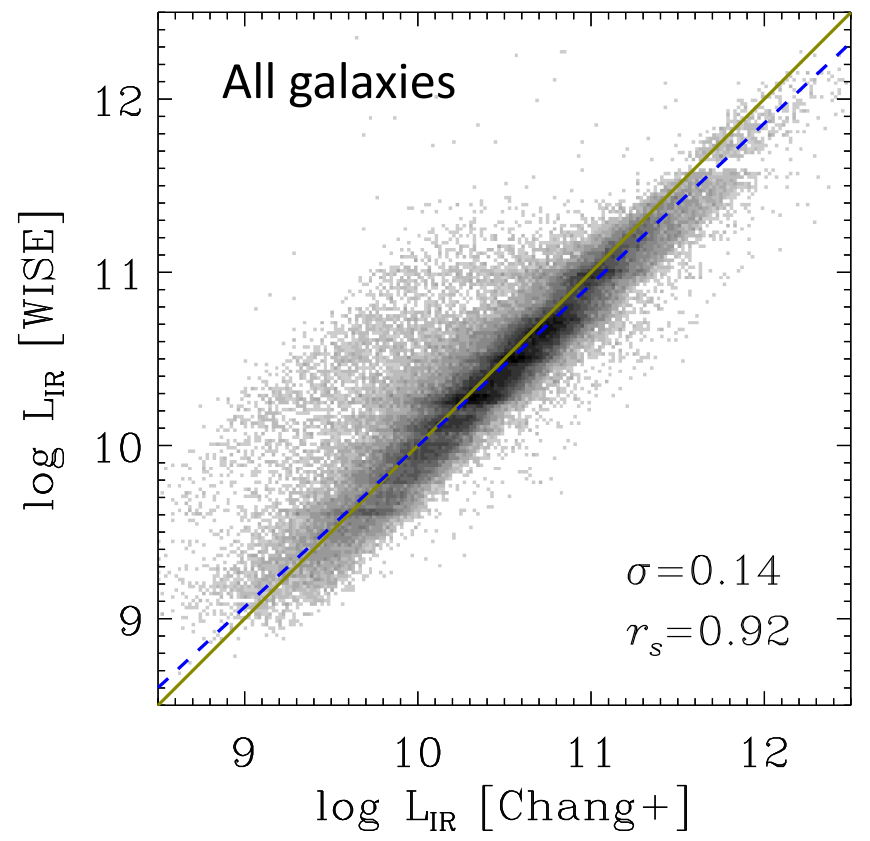

Figure 15. Comparison of total IR luminosities derived in GSWLC from WISE to total IR luminosities derived from optical/mid-IR SED fitting from Chang et al. (2015). The comparison is generally quite good. A tail to the left is due to a small number of passive galaxies for which Chang et al. luminosities are too high.

compare it to the IR luminosity that we derive from WISE $22 \mu \mathrm{m}$. There is a good overall agreement, and the scatter is smaller than in the comparison involving Ellison et al. (2016) $L_{\mathrm{IR}}$, no doubt because WISE and Chang et al. IR luminosities are somewhat correlated through the use of the same $22 \mu \mathrm{m}$ photometry. There is, however, a small fraction (1.8\%) of galaxies for which there is a $\sim 1$ dex discrepancy in $L_{\mathrm{IR}}$. These galaxies produce a bump in the distribution of specific luminosities $\left(\log \left(L_{\mathrm{IR}} / M_{*}\right)\right)$ when using Chang et al. (2015) IR luminosities, but not with WISE values. Most of these galaxies do not have $L_{\mathrm{IR}}$ from the Ellison et al. (2016) catalog, but when they do, they agree with our IR luminosity from WISE. Finally, the direct comparison of Chang et al. IR luminosities with IR luminosities from Herschel (plot not shown) confirms that Chang et al. IR luminosities are underestimated when they are based on $12 \mu \mathrm{m}$ detection without the $22 \mu \mathrm{m}$ detection.

\section{SUMMARY}

The paper presents GSWLC, an extensive catalog of physical parameters (SFRs, dust attenuations, and stellar masses) of $\sim 700,000$ SDSS galaxies covered by GALEX ( $90 \%$ of SDSS). SFRs and stellar masses were derived using state-of-the art SED fitting of UV and optical fluxes. SFRs derived independently from $22 \mu \mathrm{m}$ WISE photometry are also included in the catalog.

The construction of the catalog, the internal checks, and the comparisons with previously published catalogs have produced a number of results, which we summarize here:

1. The principal source of bias in GALEX UV photometry is from blending of unresolved sources. We provide an empirical recipe to reduce its effect.

2. Total IR luminosities obtained from WISE $22 \mu \mathrm{m}$ observations via Chary \& Elbaz (2001) luminosity- dependent templates agree remarkably well ( $\Delta<0.01$ dex, 0.07 dex of scatter) with IR luminosities obtained from WISE $22 \mu \mathrm{m}$ and Herschel-SPIRE submillimeter bands.

3. The comparison of SED sSFRs with sSFRs from the midIR and, separately, with $\mathrm{H} \alpha$ sSFRs suggests that the majority of galaxies require a dust attenuation curve that is significantly steeper than the Calzetti et al. (2000) curve and is on average similar to the Conroy et al. (2010a) curve. Allowing this steep attenuation curve to include a UV bump further improves the quality of UV/ optical SED fits.

4. Not accounting for the emission lines in the SED modeling of broadband UV-optical photometry produces significant biases in the derived (s)SFRs: up to $0.5 \mathrm{dex}$, on average, for high-sSFR galaxies (log sSFR $>-9.5)$. Stellar masses are not affected by emission lines.

5. SFRs and stellar masses are not very different $(<0.1 \mathrm{dex})$ when assuming a smooth, delayed SF history as opposed to the two-component (old and new) exponentially declining SF history, adopted for GSWLC. Fixing the old component to have started in the early universe $\left(t_{0}=10 \mathrm{Gyr}\right)$, as done here, yields 0.1 dex higher masses for galaxies with active SF, possibly removing the "outshining" bias.

6. For actively star-forming ("main") sequence galaxies $(\log$ sSFR $>-10.5)$, there is a good general agreement between SED-fitting and mid-IR (s)SFRs from GSWLC and also with (s)SFRs from the literature.

7. Aperture-corrected (total) SFRs from the MPA/JHU catalog (Brinchmann et al. 2004) have no systematics at high SFRs when compared with any other SFR, in contrast to systematic offsets reported in integral field spectroscopy studies. GSWLC, by offering two independent SFRs, can potentially be used in the forthcoming integral field spectroscopy studies to elucidate the source of the discrepancy.

8. IR luminosities of galaxies that host AGNs (as identified from the BPT diagram) and have relatively high sSFRs appear to have an excess IR emission, presumably due to AGN dust heating affecting the $22 \mu \mathrm{m}$ flux. If interpreted as SFR, this excess IR luminosity leads to sSFR overestimates of 0.2-0.6 dex, with greater effect at higher SSFR.

9. For galaxies that lie below the star-forming main sequence $\left(12<\log \mathrm{sSFR}_{\mathrm{SED}}<-11\right)$, which includes quenching, nearly quiescent, or rejuvenated galaxies, sSFRs are low and their determination is challenging by any method. Using simple (fixed factor) conversions of IR luminosity to SFR (intended for use with actively starforming galaxies) produces greatly exaggerated (s)SFRs (up to 2 dex). (s)SFRs from the UV/optical SED fitting tend to retain sensitivity in this regime. Below $\log \mathrm{sSFR}=-11.7 \quad$ (for GSWLC-M; $=-11.5$ for GSWLC-A; -12.0 for GSWLC-D), even the UV/optical sSFRs should be considered as upper limits, as these are, for all practical purposes, truly quiescent galaxies.

SDSS is the workhorse data set for many galaxy evolution studies at low redshift, but without surveys in other wavelengths it has been limited in terms of characterizing the SF. We hope that GSWLC, by combining SDSS with GALEX 
and WISE, will fill this gap and serve as a resource for many new discoveries.

The construction of GSWLC was funded through NASA ADAP award NNX12AE06G. We thank Sara Ellison for valuable comments. S.S. thanks the members of the GALEX science team for their contributions over the years, especially Tim Heckman, Chris Martin, R. Michael Rich, and Mark Seibert. Funding for SDSS-III has been provided by the Alfred P. Sloan Foundation, the Participating Institutions, the National Science Foundation, and the U.S. Department of Energy Office of Science. The SDSS-III website is http://www.sdss3.org/. Based on observations made with the NASA Galaxy Evolution Explorer. GALEX is operated for NASA by the California Institute of Technology under NASA contract NAS5-98034. This publication makes use of data products from the Widefield Infrared Survey Explorer, which is a joint project of the University of California, Los Angeles, and the Jet Propulsion Laboratory/California Institute of Technology, funded by the National Aeronautics and Space Administration.

\section{REFERENCES}

Abazajian, K., Adelman-McCarthy, J. K., Agüeros, M. A., et al. 2004, AJ, 128,502

Abazajian, K. N., Adelman-McCarthy, J. K., Agüeros, M. A., et al. 2009, ApJS, 182, 543

Ahn, C. P., Alexandroff, R., Allende Prieto, C., et al. 2014, ApJS, 211, 17

Allen, J. T., Croom, S. M., Konstantopoulos, I. S., et al. 2015, MNRAS, 446, 1567

Aloisi, A., Clementini, G., Tosi, M., et al. 2007, ApJL, 667, L151

Baldwin, J. A., Phillips, M. M., \& Terlevich, R. 1981, PASP, 93, 5

Bell, E. F., \& de Jong, R. S. 2001, ApJ, 550, 212

Bernardi, M., Shankar, F., Hyde, J. B., et al. 2010, MNRAS, 404, 2087

Bertin, E., \& Arnouts, S. 1996, A\&AS, 117, 393

Boquien, M., Buat, V., \& Perret, V. 2014, A\&A, 571, A72

Boquien, M., Kennicutt, R., Calzetti, D., et al. 2016, A\&A, 591, A6

Bothun, G. D. 1982, ApJS, 50, 39

Bressan, A., Aussel, H., Granato, G. L., et al. 2001, ApSSS, 277, 251

Brinchmann, J., Charlot, S., White, S. D. M., et al. 2004, MNRAS, 351, 115

Bruzual, A. G. 1983, ApJ, 273, 105

Bruzual, A. G., \& Charlot, S. 1993, ApJ, 405, 538

Bruzual, G., \& Charlot, S. 2003, MNRAS, 344, 1000

Buat, V., Giovannoli, E., Takeuchi, T. T., et al. 2011, A\&A, 529, A22

Buat, V., \& Xu, C. 1996, A\&A, 306, 61

Budavári, T., Heinis, S., Szalay, A. S., et al. 2009, ApJ, 694, 1281

Calzetti, D. 2013, Secular Evolution of Galaxies (Cambridge: Cambridge Univ. Press)

Calzetti, D., Armus, L., Bohlin, R. C., et al. 2000, ApJ, 533, 682

Cardelli, J. A., Clayton, G. C., \& Mathis, J. S. 1989, ApJ, 345, 245

Chabrier, G. 2003, PASP, 115, 763

Chang, Y.-Y., van der Wel, A., da Cunha, E., \& Rix, H.-W. 2015, ApJS, 219, 8

Charlot, S., \& Bruzual, A. G. 1991, ApJ, 367, 126

Charlot, S., \& Fall, S. M. 2000, ApJ, 539, 718

Chary, R., \& Elbaz, D. 2001, ApJ, 556, 562

Cid Fernandes, R., Mateus, A., Sodré, L., Stasińska, G., \& Gomes, J. M. 2005, MNRAS, 358, 363

Conroy, C. 2013, ARA\&A, 51, 393

Conroy, C., \& Gunn, J. E. 2010, ApJ, 712, 833

Conroy, C., Gunn, J. E., \& White, M. 2009, ApJ, 699, 486

Conroy, C., Schiminovich, D., \& Blanton, M. R. 2010a, ApJ, 718, 184

Conroy, C., White, M., \& Gunn, J. E. 2010b, ApJ, 708, 58

Cortese, L., Boselli, A., Franzetti, P., et al. 2008, MNRAS, 386, 1157

Courteau, S., Cappellari, M., de Jong, R. S., et al. 2014, RvMP, 86, 47

da Cunha, E., Charlot, S., \& Elbaz, D. 2008, MNRAS, 388, 1595

Dale, D. A., \& Helou, G. 2002, ApJ, 576, 159

Donoso, E., Yan, L., Tsai, C., et al. 2012, ApJ, 748, 80

Dopita, M. A., Kewley, L. J., Heisler, C. A., \& Sutherland, R. S. 2000, ApJ, 542,224

Dressler, A., \& Gunn, J. E. 1983, ApJ, 270, 7
Ellison, S. L., Teimoorinia, H., Rosario, D. J., \& Mendel, J. T. 2016, MNRAS, 455,370

Faber, S. M. 1972, A\&A, 20, 361

Fang, J. J., Faber, S. M., Salim, S., Graves, G. J., \& Rich, R. M. 2012, ApJ, 761,23

Fitzpatrick, E. L., \& Massa, D. 1986, ApJ, 307, 286

Gallazzi, A., Charlot, S., Brinchmann, J., White, S. D. M., \& Tremonti, C. A. 2005, MNRAS, 362, 41

Gavazzi, G., Bonfanti, C., Sanvito, G., Boselli, A., \& Scodeggio, M. 2002, ApJ, 576, 135

Goto, T. 2007, MNRAS, 381, 187

Guiderdoni, B., \& Rocca-Volmerange, B. 1987, A\&A, 186, 1

Hao, C.-N., Kennicutt, R. C., Johnson, B. D., et al. 2011, ApJ, 741, 124

Kauffmann, G., Heckman, T. M., Budavári, T., et al. 2007, ApJS, 173, 357

Kauffmann, G., Heckman, T. M., Tremonti, C., et al. 2003a, MNRAS, 346, 1055

Kauffmann, G., Heckman, T. M., White, S. D. M., et al. 2003b, MNRAS, 341,33

Kennicutt, R. C., Jr. 1998, ARA\&A, 36, 189

Kewley, L. J., Groves, B., Kauffmann, G., \& Heckman, T. 2006, MNRAS, 372, 961

Kriek, M., Labbé, I., Conroy, C., et al. 2010, ApJL, 722, L64

Lang, D., Hogg, D. W., \& Schlegel, D. J. 2016, AJ, 151, 36

Larson, R. B., \& Tinsley, B. M. 1978, ApJ, 219, 46

Liang, Y. C., Yin, S. Y., Hammer, F., et al. 2006, ApJ, 652, 257

Maraston, C. 2005, MNRAS, 362, 799

Maraston, C. 2011, in ASP Conf. Ser. 445, Why Galaxies Care about AGB Stars, II., ed. F. Kerschbaum, T. Lebzelter, \& R. F. Wing (San Francisco: ASP), 391

Marcillac, D., Elbaz, D., Chary, R. R., et al. 2006, A\&A, 451, 57

Marigo, P. 2015, in ASP Conf. Ser. 497, Why Galaxies Care about AGB Stars, III., ed. F. Kerschbaum, R. F. Wing, \& J. Hron (San Francisco, CA: ASP), 229

Martin, D. C., Seibert, M., Buat, V., et al. 2005, ApJL, 619, L59

McGaugh, S. S., \& Schombert, J. M. 2014, AJ, 148, 77

Meert, A., Vikram, V., \& Bernardi, M. 2016, MNRAS, 455, 2440

Mendel, J. T., Simard, L., Palmer, M., Ellison, S. L., \& Patton, D. R. 2014 ApJS, 210, 3

Michałowski, M. J., Hayward, C. C., Dunlop, J. S., et al. 2014, A\&A, 571, A75

Mitchell, P. D., Lacey, C. G., Baugh, C. M., \& Cole, S. 2013, MNRAS, 435, 87

Morrissey, P., Conrow, T., Barlow, T. A., et al. 2007, ApJS, 173, 682

Mullaney, J. R., Alexander, D. M., Goulding, A. D., \& Hickox, R. C. 2011, MNRAS, 414, 1082

Muzzin, A., van Dokkum, P., Kriek, M., et al. 2010, ApJ, 725, 742

Noll, S., Burgarella, D., Giovannoli, E., et al. 2009, A\&A, 507, 1793

Pace, C., \& Salim, S. 2016, ApJ, 818, 65

Pacifici, C., da Cunha, E., Charlot, S., et al. 2015, MNRAS, 447, 786

Pacifici, C., Kassin, S. A., Weiner, B., Charlot, S., \& Gardner, J. P. 2013 , ApJL, 762, L15

Papovich, C., Dickinson, M., \& Ferguson, H. C. 2001, ApJ, 559, 620

Peek, J. E. G., \& Schiminovich, D. 2013, ApJ, 771, 68

Renzini, A., \& Buzzoni, A. 1986, Spectral Evolution of Galaxies, Vol. 122 (Dordrecht: Reidel), 189

Richards, S. N., Bryant, J. J., Croom, S. M., et al. 2016, MNRAS, 455, 2826

Rosario, D. J., Mendel, J. T., Ellison, S. L., Lutz, D., \& Trump, J. R. 2016 , MNRAS, 457, 2703

Salim, S., Charlot, S., Rich, R. M., et al. 2005, ApJL, 619, L39

Salim, S., Dickinson, M., Michael Rich, R., et al. 2009, ApJ, 700, 161

Salim, S., Fang, J. J., Rich, R. M., Faber, S. M., \& Thilker, D. A. 2012, ApJ, 755,105

Salim, S., Rich, R. M., Charlot, S., et al. 2007, ApJS, 173, 267

Salmon, B., Papovich, C., Long, J., et al. 2015, ApJ, 827, 20

Sanders, D. B., \& Mirabel, I. F. 1996, ARA\&A, 34, 749

Sawicki, M., \& Yee, H. K. C. 1998, AJ, 115, 1329

Schlegel, D. J., Finkbeiner, D. P., \& Davis, M. 1998, ApJ, 500, 525

Searle, L., Sargent, W. L. W., \& Bagnuolo, W. G. 1973, ApJ, 179, 427

Shapley, A. E., Steidel, C. C., Erb, D. K., et al. 2005, ApJ, 626, 698

Simard, L., Mendel, J. T., Patton, D. R., Ellison, S. L., \& McConnachie, A. W. 2011, ApJS, 196, 11

Simha, V., Weinberg, D. H., Conroy, C., et al. 2014, arXiv:1404.0402

Smith, D. J. B., \& Hayward, C. C. 2015, MNRAS, 453, 1597

Sorba, R., \& Sawicki, M. 2015, MNRAS, 452, 235

Stasińska, G., Vale Asari, N., Cid Fernandes, R., et al. 2008, MNRAS, 391, L29

Stecher, T. P. 1965, ApJ, 142, 1683 
Strauss, M. A., Weinberg, D. H., Lupton, R. H., et al. 2002, AJ, 124, 1810

Taylor, E. N., Hopkins, A. M., Baldry, I. K., et al. 2011, MNRAS, 418, 1587 Tinsley, B. M., \& Gunn, J. E. 1976, ApJ, 203, 52

Tremonti, C. A., Heckman, T. M., Kauffmann, G., et al. 2004, ApJ, 613, 898

Tully, R. B., Courtois, H. M., \& Sorce, J. G. 2016, AJ, 152, 50

Tully, R. B., Mould, J. R., \& Aaronson, M. 1982, ApJ, 257, 527

van der Wel, A., Franx, M., Wuyts, S., et al. 2006, ApJ, 652, 97

Villaume, A., Conroy, C., \& Johnson, B. D. 2015, ApJ, 806, 82

Walcher, J., Groves, B., Budavári, T., \& Dale, D. 2011, Ap\&SS, 331, 1
Weisz, D. R., Dalcanton, J. J., Williams, B. F., et al. 2011, ApJ, 739, 5

West, A. A., Garcia-Appadoo, D. A., Dalcanton, J. J., et al. 2010, AJ, 139, 315

Wild, V., Charlot, S., Brinchmann, J., et al. 2011, MNRAS, 417, 1760

Wright, E. L., Eisenhardt, P. R. M., Mainzer, A. K., et al. 2010, AJ, 140, 1868

Yan, L., Donoso, E., Tsai, C.-W., et al. 2013, AJ, 145, 55

Yang, Y., Zabludoff, A. I., Zaritsky, D., \& Mihos, J. C. 2008, ApJ, 688, 945

Yuan, H. B., Liu, X. W., \& Xiang, M. S. 2013, MNRAS, 430, 2188

Zibetti, S., Gallazzi, A., Charlot, S., Pierini, D., \& Pasquali, A. 2013, MNRAS, 428, 1479 